\title{
Nonlinear viscous liquid jets from a rotating orifice
}

\author{
E.I. PĂRĂU ${ }^{2}$, S.P DECENT ${ }^{1}$, M.J.H. SIMMONS ${ }^{3}$, D.C.Y. WONG ${ }^{3}$, \\ A.C. $\mathrm{KING}^{1}$ \\ ${ }^{1}$ School of Mathematics, The University of Birmingham, Edgbaston, Birmingham, \\ B15 2TT, UK \\ ${ }^{2}$ School of Mathematics, University of East Anglia, Norwich,NR4 7TJ, UK \\ ${ }^{3}$ School of Engineering (Chemical Engineering), The University of Birmingham, \\ Edgbaston, Birmingham, B15 2TT, UK \\ email:e.parau@uea.ac.uk,decentsp@for.mat.bham.ac.uk
}

\begin{abstract}
A liquid jet follows a curved trajectory when the orifice from which the jet emerges is rotating. Surface tension driven instabilities cause the jet to lose coherence and break to form droplets. The sizes of the drops formed from such jets are in general not uniform, ranging from drops with diameters of the order of the jet diameter to droplets with diameters which are several orders of magnitude smaller. This presentation details a theoretical investigation of the effects of changing operating parameters on the break-up of curved liquid jets in stagnant air at room temperature and pressure. The Navier-Stokes equations are solved in this system with the usual viscous free surface boundary conditions, using an asymptotic method based upon a slender jet assumption, which is clearly appropriate from experimental observations of the jet. We also present nonlinear temporal simulations of the breakup of the liquid jets using our slender theory. These simulations based upon both a steady trajectory assumption, and the more general equations which allow for an unsteady trajectory, show all the breakup modes viewed in experiments. Satellite droplet formation is also considered.
\end{abstract}

Keywords: viscosity, rotation, jet, unsteady

\section{Introduction}

In Wallwork et al. [1] we presented asymptotic and numerical results for an inviscid liquid emerging from a rapidly rotating container. Also, experimental results were given for a low viscosity liquid, showing good agreement between theory and experimental data. This work has applications to prilling (Anderson \& Yttri [2]) which is a common industrial technique for producing pellets (for example, fertiliser and also magnesium pellets). In this process thousands of liquid jets emerge from a rapidly rotating drum. Each jet is curved due to the rotational forces on it, and each jet breaks up into droplets due to a surface tension driven instability. These droplets cool and solidify forming pellets. There are numerous economic reasons for wanting to control this instability process (including the optimization of the process, the uniformity of the product, and the minimization of satellite drop formation to decrease 
waste), and no progress can be made on this front until a thorough understanding of the instability has been achieved.

Previous work presented on this prilling scenario has examined inviscid liquids (Wallwork et al. [1], Decent et al. [3], Părău et al. [4]). In industrial settings, the liquids used are viscous. We concentrate on the Newtonian model for viscosity here due to the complexity of the equations produced even in this situation, though non-Newtonian models are of considerable engineering interest and is the subject of current work. A series of experiments was performed to see how altering various parameters affects trajectory, stability and droplet formation of the jet. A detailed description of these experiments carried out in the School of Chemical Engineering, University of Birmingham, is given in Wong et al. [31]. A stability analysis for a viscous jet was performed by Decent et al. [6].

One of the first to study the capillary break-up of axisymmetric inviscid liquid jets was Rayleigh [7]. Rayleigh [8] and Weber [9] incorporated viscosity into the linear instability calculation for a straight jet. Nonlinear one-dimensional models for axisymmetric inviscid jets have been developed, by assuming a periodic disturbance along the infinite jet, by many authors (see Lee [10], Mansour and Lundgren [11], Schulkes [12], Papageorgiou and Orellana [13]). The presence of the orifice has also been included, first by Keller et al. [14], Pimbley and Lee [15], and by Bogy $[16,17]$ in the context of the one-dimensional Cosserat theory. There are more recent jet simulations, which consider the jet having a finite length (see Eggers and Dupont [18], Hilbing and Heister [19], Cheong and Howes [20]). An extensive review of the work on axisymmetric liquid jets is given by Eggers [21]. Curved liquid jets and sheets have been studied before by Weber [9], Vanden-Broeck and Keller [22], Dias and Vanden-Broeck [23], Finnicum et al. [24], Cummings and Howell [25], Entov and Yarin [26], Yarin [27] and others.

In this paper, the equation of motion are presented in Section 2 and an asymptotic analysis is performed in Section 3, in order to obtain a coupled partial differential equation system at leading order. In Section 4 a numerical method is presented to solve the leading order equations for steady-state solutions. Typical nonlinear temporal results, showing the influence of various parameters, are presented in Section 5 . The more general equations, when the centreline is time-dependent, are obtained in Section 6 and some conclusions are made in Section 7. 


\section{The equations of motion}

We consider a circular cylindrical container of radius $s_{0}$ rotating about its axis with rotation rate $\Omega$. A viscous jet emerges from an orifice on the surface of the cylinder.

We derive non-dimensional equations of motion following Wallwork et al. [1] but incorporating viscosity. We work in a rotating reference frame in which the orifice is fixed, we switch off gravity (so that we assume rotational forces are much greater than gravitational ones, as in prilling) and we use a curvilinear coordinate system $(s, n, \phi)$ where $s$ is the arclength along the centreline of the jet from the orifice, and $(n, \phi)$ are plane polar coordinates in any cross-section of the jet. The centreline of the jet is at $(X, 0, Z)$ in Cartesian coordinates with the origin at the centre of the orifice, where the $x$-axis is directed normal to the surface of the container in the initial direction of the jet and the $z$-axis is orthogonal to the $x$-axis in the plane of the centreline of the jet. The positive $z$-axis points in the opposite direction to the motion of the container. Also, $X=X(s, t)$ and $Z=Z(s, t)$ where $t$ is time.

We show in detail only the deduction of the boundary conditions: more detail is given in Wallwork [5]. Following Batchelor [28], p. 600, the components of the stress tensor $\sigma_{i j}$ in the orthogonal curvilinear coordinate system are:

$$
\begin{aligned}
& \sigma_{11}=\frac{1}{h_{1}} \frac{\partial u_{1}}{\partial \xi_{1}}+\frac{u_{2}}{h_{1} h_{2}} \frac{\partial h_{1}}{\partial \xi_{2}}+\frac{u_{3}}{h_{3} h_{2}} \frac{\partial h_{1}}{\partial \xi_{3}}, \\
& \sigma_{23}=\frac{h_{3}}{2 h_{2}} \frac{\partial}{\partial \xi_{2}}\left(\frac{u_{3}}{h_{3}}\right)+\frac{h_{2}}{2 h_{3}} \frac{\partial}{\partial \xi_{3}}\left(\frac{u_{2}}{h_{2}}\right),
\end{aligned}
$$

and the four other expressions are obtained by cyclic interchange of suffixes. In our case $\xi_{1}=s, \xi_{2}=n, \xi_{3}=\phi$, and $h_{1}=h_{s}=1+$ $n \cos \phi\left(X_{s} Z_{s s}-X_{s s} Z_{s}\right), h_{2}=1, h_{3}=n$, (see [1]) so

$$
\begin{array}{rcc}
\sigma_{s s} & =-p+2 \mu \cdot \frac{1}{h_{s}} \cdot\left[\frac{\partial u}{\partial s}+(v \cos \phi-w \sin \phi)\left(X_{s} Z_{s s}-X_{s s} Z_{s}\right)\right], \\
\sigma_{n n} & = & -p+2 \mu \frac{\partial v}{\partial n}, \\
\sigma_{\phi \phi} & = & -p+2 \mu \cdot \frac{1}{n} \cdot\left(\frac{\partial w}{\partial \phi}+v\right), \\
\sigma_{s n}=\sigma_{n s} & \quad & \mu\left[\frac{1}{h_{s}} \frac{\partial v}{\partial s}+\frac{\partial u}{\partial n}-\frac{u}{h_{s}} \cos \phi\left(X_{s} Z_{s s}-X_{s s} Z_{s}\right)\right], \\
\sigma_{n \phi}=\sigma_{\phi n} & = & \mu\left(\frac{\partial w}{\partial n}-\frac{w}{n}+\frac{1}{n} \cdot \frac{\partial v}{\partial \phi}\right), \\
\sigma_{s \phi}=\sigma_{\phi s} & = & \mu\left[\frac{1}{n} \cdot \frac{\partial u}{\partial \phi}+\frac{u}{h_{s}} \sin \phi\left(X_{s} Z_{s s}-X_{s s} Z_{s}\right)+\frac{1}{h_{s}} \cdot \frac{\partial w}{\partial s}\right],
\end{array}
$$


where the liquid velocity is $u \mathbf{e}_{\mathbf{s}}+v \mathbf{e}_{\mathbf{n}}+w \mathbf{e}_{\phi}, \mathbf{e}_{\mathbf{s}}, \mathbf{e}_{\mathbf{n}}$ and $\mathbf{e}_{\phi}$ are the unit vectors tangential, normal and azimuthal to the jet's centreline respectively, defined in [1]. The position of the free-surface is given by $n=R(s, \phi, t)$. The pressure is $p$ and the viscosity is $\mu$.

The unit normal vector to the surface of the jet $n-R(s, \phi, t)=0$ is

$$
\mathbf{n}=\frac{1}{E}\left(-\frac{\partial R}{\partial s} \cdot \frac{1}{h_{s}} \cdot \mathbf{e}_{\mathbf{s}}+\mathbf{e}_{\mathbf{n}}-\frac{\partial R}{\partial \phi} \cdot \frac{1}{R} \cdot \mathbf{e}_{\phi}\right),
$$

and the tangent vectors, obtained as $\mathbf{t}_{\mathbf{1}}=\frac{\partial \mathbf{r}_{\mathbf{P}}}{\partial s}$ and $\mathbf{t}_{\mathbf{2}}=\frac{\partial \mathbf{r}_{\mathbf{P}}}{\partial \phi}$ (where $\mathbf{r}_{\mathbf{P}}$ is the position vector of a particle $\mathbf{P}$ on the free surface):

$$
\mathbf{t}_{\mathbf{1}}=\mathbf{e}_{\mathbf{s}}+\frac{\partial R}{\partial s} \cdot \frac{1}{h_{s}} \cdot \mathbf{e}_{\mathbf{n}}, \quad \text { and } \mathbf{t}_{\mathbf{2}}=\frac{\partial R}{\partial \phi} \cdot \frac{1}{R} \cdot \mathbf{e}_{\mathbf{n}}+\mathbf{e}_{\phi},
$$

where

$$
E=\left(1+\left(\frac{\partial R}{\partial s}\right)^{2} \cdot \frac{1}{h_{s}^{2}}+\left(\frac{\partial R}{\partial \phi}\right)^{2} \cdot \frac{1}{R^{2}}\right)^{1 / 2}
$$

The normal stress condition is $\mathbf{n} \cdot \underline{\sigma} \cdot \mathbf{n}=\sigma \kappa$, where $\kappa$ is given by

$$
\kappa=\frac{1}{n h_{s}}\left(\frac{\partial}{\partial s}\left(\frac{-\frac{n}{h_{s}} \frac{\partial R}{\partial s}}{E}\right)+\frac{\partial}{\partial n}\left(\frac{n h_{s}}{E}\right)+\frac{\partial}{\partial \phi}\left(\frac{-\frac{h_{s}}{n} \frac{\partial R}{\partial \phi}}{E}\right)\right),
$$

for $n=R(s, \phi, t)$ and the surface tension is $\sigma$. The tangential stress condition(s) are $\mathbf{t}_{\mathbf{i}} \cdot \underline{\sigma} \cdot \mathbf{n}, i=1,2$.

After some algebraic manipulations, the normal stress condition can be written as

$$
\begin{array}{r}
p-2 \frac{\mu}{E^{2}}\left\{\left(\frac{\partial R}{\partial s}\right)^{2} \frac{1}{h_{s}^{3}}\left[\frac{\partial u}{\partial s}+(v \cos \phi-w \sin \phi)\left(X_{s} Z_{s s}-X_{s s} Z_{s}\right)\right]\right. \\
+\frac{\partial v}{\partial n}+\left(\frac{\partial R}{\partial \phi}\right)^{2} \frac{1}{R^{3}}\left(\frac{\partial w}{\partial \phi}+v\right)-\frac{\partial R}{\partial s} \cdot \frac{1}{h_{s}}\left[\frac{\partial v}{\partial s} \frac{1}{h_{s}}+\frac{\partial u}{\partial n}\right. \\
\left.-\frac{u}{h_{s}} \cos \phi\left(X_{s} Z_{s s}-X_{s s} Z_{s}\right)\right]-\frac{\partial R}{\partial \phi} \frac{1}{R}\left(\frac{\partial w}{\partial n}-\frac{w}{R}+\frac{\partial v}{\partial \phi} \frac{1}{R}\right) \\
\left.+\frac{\partial R}{\partial s} \frac{\partial R}{\partial \phi} \frac{1}{h_{s}} \frac{1}{R}\left[\frac{\partial u}{\partial \phi} \frac{1}{R}+\frac{u}{h_{s}} \sin \phi\left(X_{s} Z_{s s}-X_{s s} Z_{s}\right)+\frac{\partial u}{\partial s} \frac{1}{h_{s}}\right]\right\}=\sigma \kappa .
\end{array}
$$

The first tangential stress condition is

$$
\begin{array}{r}
\left(1-\left(\frac{\partial R}{\partial s}\right)^{2} \frac{1}{h_{s}^{2}}\right)\left[\frac{\partial v}{\partial s} \frac{1}{h_{s}}+\frac{\partial u}{\partial n}-\frac{u}{h_{s}} \cos \phi\left(X_{s} Z_{s s}-X_{s s} Z_{s}\right)\right] \\
+2 \frac{\partial R}{\partial s} \frac{1}{h_{s}}\left[\frac{\partial v}{\partial n}-\frac{\partial u}{\partial s} \frac{1}{h_{s}}-\frac{v \cos \phi-w \sin \phi}{h_{s}}\left(X_{s} Z_{s s}-X_{s s} Z_{s}\right)\right]=0,
\end{array}
$$


and the second tangential stress condition, corresponding to stress in the $\phi$ direction around the jet is

$$
\begin{aligned}
&(1\left.-\left(\frac{\partial R}{\partial \phi}\right)^{2} \frac{1}{R^{2}}\right)\left(\frac{\partial w}{\partial n}-\frac{w}{R}+\frac{\partial v}{\partial \phi} \frac{1}{R}\right) \\
&+2 \frac{\partial R}{\partial \phi} \frac{1}{R}\left(\frac{\partial v}{\partial n}-\frac{1}{R}\left(\frac{\partial w}{\partial \phi}+v\right)\right)=0
\end{aligned}
$$

As an observation, for an axisymmetric jet without azimuthal velocity the normal stress condition becomes

$$
p-2 \frac{\mu}{E_{1}^{2}}\left[\left(\frac{\partial R}{\partial s}\right)^{2} \frac{\partial u}{\partial s}+\frac{\partial v}{\partial n}-\frac{\partial R}{\partial s}\left(\frac{\partial v}{\partial s}+\frac{\partial u}{\partial n}\right)\right]=\sigma \kappa_{1},
$$

where $E_{1}=\left(1+\left(\frac{\partial R}{\partial s}\right)^{2}\right)^{1 / 2}$

$$
\text { and } \kappa_{1}=\frac{1}{n}\left(\frac{\partial}{\partial s}\left(\frac{-n \frac{\partial R}{\partial s}}{E_{1}}\right)+\frac{\partial}{\partial n}\left(\frac{n}{E_{1}}\right)\right)=-\frac{\partial}{\partial s}\left(\frac{\frac{\partial R}{\partial s}}{E_{1}}\right)+\frac{1}{R E_{1}} \text {, }
$$

and the tangential stress condition is

$$
\left(1-\left(\frac{\partial R}{\partial s}\right)^{2}\right)\left(\frac{\partial v}{\partial s}+\frac{\partial u}{\partial n}\right)+2 \frac{\partial R}{\partial s}\left(\frac{\partial v}{\partial n}-\frac{\partial u}{\partial s}\right)=0
$$

which are exactly the boundary condition found by Eggers \& Dupont [18], Garcia \& Castellanos [29] and others.

The unit vectors are

$$
\begin{gathered}
\mathbf{e}_{s}=X_{s} \cdot \mathbf{i}+Z_{s} \cdot \mathbf{k} \\
\mathbf{e}_{n}=\cos \phi Z_{s} \cdot \mathbf{i}-\sin \phi \cdot \mathbf{j}-\cos \phi X_{s} \cdot \mathbf{k} \\
\mathbf{e}_{\phi}=-\sin \phi Z_{s} \cdot \mathbf{i}-\cos \phi \cdot \mathbf{j}+\sin \phi X_{s} \cdot \mathbf{k},
\end{gathered}
$$

the position vector is

$\mathbf{r}:=\int_{0}^{s} \mathbf{e}_{s} d s+n \mathbf{e}_{n}=\left(X+n \cos \phi Z_{s}\right) \cdot \mathbf{i}-n \sin \phi \cdot \mathbf{j}+\left(Z-n \cos \phi X_{s}\right) \cdot \mathbf{k}$, so the velocity field is

$$
\frac{d \mathbf{r}}{d t}=u \mathbf{e}_{s}+v \mathbf{e}_{n}+w \mathbf{e}_{\phi}
$$


On the other hand we have

$$
\begin{gathered}
\frac{d \mathbf{r}}{d t}=\left[X_{s} \frac{\partial s}{\partial t}+X_{t}+\frac{\partial n}{\partial t} \cos \phi Z_{s}-\frac{\partial \phi}{\partial t} n \sin \phi Z_{s}+n \cos \phi Z_{s s} \frac{\partial s}{\partial t}+n \cos \phi Z_{s t}\right] \cdot \mathbf{i} \\
-\left[\frac{\partial n}{\partial t} \sin \phi+\frac{\partial \phi}{\partial t} n \cos \phi\right] \cdot \mathbf{j} \\
+\left[Z_{s} \frac{\partial s}{\partial t}+Z_{t}-\frac{\partial n}{\partial t} \cos \phi X_{s}+\frac{\partial \phi}{\partial t} n \sin \phi X_{s}-n \cos \phi X_{s s} \frac{\partial s}{\partial t}-n \cos \phi X_{s t}\right] \cdot \mathbf{k}
\end{gathered}
$$

and

$u \mathbf{e}_{s}+v \mathbf{e}_{n}+w \mathbf{e}_{\phi}=\left[u X_{s}+v \cos \phi Z_{s}-w \sin \phi Z_{s}\right] \cdot \mathbf{i}+[-v \sin \phi-w \cos \phi] \cdot \mathbf{j}$

$$
+\left[u Z_{s}-v \cos \phi X_{s}+w \sin \phi X_{s}\right] \cdot \mathbf{k},
$$

so

$$
\begin{gathered}
u=\frac{\partial s}{\partial t}\left(1+n \cos \phi\left(X_{s} Z_{s s}-X_{s s} Z_{s}\right)\right)+X_{t} X_{s}+Z_{t} Z_{s}+n \cos \phi\left(X_{s} Z_{s t}-X_{s t} Z_{s}\right) \\
v=\frac{\partial n}{\partial t}+\cos \phi\left(X_{t} Z_{s}-X_{s} Z_{t}\right) \\
w=n \frac{\partial \phi}{\partial t}-\sin \phi\left(X_{t} Z_{s}-X_{s} Z_{t}\right)
\end{gathered}
$$

The kinematic condition is

$$
\frac{D}{D t}(R(s, \phi, t)-n)=0 \quad \text { for } n=R(s, \phi, t)
$$

or

$$
\frac{\partial R}{\partial t}+\frac{\partial R}{\partial s} \frac{\partial s}{\partial t}+\frac{\partial R}{\partial \phi} \frac{\partial \phi}{\partial t}-\frac{\partial n}{\partial t}=0
$$

which gives us, after using the relations (4),

$$
\begin{gathered}
\left(1+n \cos \phi\left(X_{s} Z_{s s}-X_{s s} Z_{s}\right)\right)\left(\frac{\partial R}{\partial t}+\cos \phi\left(X_{t} Z_{s}-X_{s} Z_{t}\right)-v+\right. \\
\left.\frac{\partial R}{\partial \phi} \frac{w}{n} \cdot+\frac{\partial R}{\partial \phi} \frac{1}{n} \sin \phi\left(X_{t} Z_{s}-X_{s} Z_{t}\right)\right)+u \frac{\partial R}{\partial s} \\
-\frac{\partial R}{\partial s}\left(X_{t} X_{s}+Z_{t} Z_{s}+n \cos \phi\left(X_{s} Z_{s s}-X_{s s} Z_{s}\right)\right)=0 .
\end{gathered}
$$

We non-dimensionalise using the transformations

$$
\begin{aligned}
\bar{u}=\frac{u}{U}, \bar{v} & =\frac{v}{U}, \bar{w}=\frac{w}{U}, \bar{p}=\frac{p}{\rho U^{2}}, \bar{n}=\frac{n}{a}, \epsilon=\frac{a}{s_{0}}, \\
\bar{R} & =\frac{R}{a}, \bar{s}=\frac{s}{s_{0}}, \bar{t}=\frac{t U}{s_{0}}, \bar{X}=\frac{X}{s_{0}}, \bar{Z}=\frac{Z}{s_{0}},
\end{aligned}
$$


where $U$ is the exit speed of the jet in the rotating frame, $\rho$ is the liquid's density, $s_{0}$ the radius of the drum, $a$ the radius of the orifice, $\epsilon$ is an aspect ratio, $p$ is the pressure, $R$ is the jet radius and $u, v$ and $w$ are the tangential, radial and azimuthal velocity components relative to the centreline of the jet respectively. The bars denote dimensionless quantities in the above expressions. Dropping overbars and using standard methods we obtain the continuity equation

$$
\begin{array}{r}
\epsilon n \frac{\partial u}{\partial s}+h_{s}\left(v+n \frac{\partial v}{\partial n}+\frac{\partial w}{\partial \phi}\right) \\
+\epsilon n\left(X_{s} Z_{s s}-X_{s s} Z_{s}\right)(v \cos \phi-w \sin \phi)=0,
\end{array}
$$

the Navier-Stokes equations

$$
\begin{aligned}
& h_{s}\left(\epsilon \frac{\partial u}{\partial t}+\epsilon(v \cos \phi-w \sin \phi)\left(Z_{s t} X_{s}-X_{s t} Z_{s}\right)+v \frac{\partial u}{\partial n}+\frac{w}{n} \frac{\partial u}{\partial \phi}\right) \\
& +\epsilon u \frac{\partial u}{\partial s}+\epsilon u\left(X_{s} Z_{s s}-X_{s s} Z_{s}\right)(v \cos \phi-w \sin \phi) \\
& =-\epsilon \frac{\partial p}{\partial s}+\left(\frac{2 \epsilon}{R b}(v \cos \phi-w \sin \phi)+\frac{\epsilon}{R b^{2}}\left((X+1) X_{s}+Z Z_{s}\right)\right) h_{s} \\
& +\frac{1}{R e} \frac{1}{\epsilon n}\left\{\frac { - \epsilon ^ { 3 } n ^ { 2 } \operatorname { c o s } \phi ( X _ { s } Z _ { s s s } - X _ { s s s } Z _ { s } ) } { h _ { s } ^ { 2 } } \left(\frac{\partial u}{\partial s}+v \cos \phi\left(X_{s} Z_{s s}-X_{s s} Z_{s}\right)\right.\right. \\
& \left.-w \sin \phi\left(X_{s} Z_{s s}-X_{s s} Z_{s}\right)\right)+\frac{\epsilon^{2} n}{h_{s}}\left(-u\left(X_{s} Z_{s s}-X_{s s} Z_{s}\right)^{2}+\frac{\partial^{2} u}{\partial s^{2}}\right. \\
& +2 \frac{\partial v}{\partial s} \cos \phi\left(X_{s} Z_{s s}-X_{s s} Z_{s}\right)+v \cos \phi\left(X_{s} Z_{s s s}-X_{s s s} Z_{s}\right) \\
& \left.-2 \frac{\partial w}{\partial s} \sin \phi\left(X_{s} Z_{s s}-X_{s s} Z_{s}\right)-w \sin \phi\left(X_{s} Z_{s s s}-X_{s s s} Z_{s}\right)\right)+ \\
& \left(1+2 \epsilon n \cos \phi\left(X_{s} Z_{s s}-X_{s s} Z_{s}\right)\right) \frac{\partial u}{\partial n}+n h_{s} \frac{\partial^{2} u}{\partial n^{2}} \\
& \left.-\epsilon \frac{\partial u}{\partial \phi} \sin \phi\left(X_{s} Z_{s s}-X_{s s} Z_{s}\right)+\frac{h_{s}}{n} \frac{\partial^{2} u}{\partial \phi^{2}}\right\} \\
& h_{s}\left(\epsilon \frac{\partial v}{\partial t}+\epsilon u \cos \phi\left(X_{s t} Z_{s}-Z_{s t} X_{s}\right)+v \frac{\partial v}{\partial n}+\frac{w}{n} \frac{\partial v}{\partial \phi}-\frac{w^{2}}{n}\right) \\
& +\epsilon u \frac{\partial v}{\partial s}-\epsilon \cos \phi\left(X_{s} Z_{s s}-X_{s s} Z_{s}\right) u^{2} \\
& \begin{array}{r}
=\left(-\frac{\partial p}{\partial n}-\frac{2 \epsilon}{R b} u \cos \phi+\frac{\epsilon \cos \phi}{R b^{2}}\left((X+1) Z_{s}-Z X_{s}+\epsilon n \cos \phi\right)\right) h_{s} \\
+\frac{1}{R e} \frac{1}{\epsilon n}\left\{\frac{-\epsilon^{3} n^{2} \cos \phi\left(X_{s} Z_{s s s}-X_{s s s} Z_{s}\right)}{h_{s}^{2}}\left(\frac{\partial v}{\partial s}-u \cos \phi\left(X_{s} Z_{s s}-X_{s s} Z_{s}\right)\right)\right.
\end{array} \\
& \begin{array}{r}
=\left(-\frac{\partial p}{\partial n}-\frac{2 \epsilon}{R b} u \cos \phi+\frac{\epsilon \cos \phi}{R b^{2}}\left((X+1) Z_{s}-Z X_{s}+\epsilon n \cos \phi\right)\right) h_{s} \\
+\frac{1}{R e} \frac{1}{\epsilon n}\left\{\frac{-\epsilon^{3} n^{2} \cos \phi\left(X_{s} Z_{s s s}-X_{s s s} Z_{s}\right)}{h_{s}^{2}}\left(\frac{\partial v}{\partial s}-u \cos \phi\left(X_{s} Z_{s s}-X_{s s} Z_{s}\right)\right)\right.
\end{array}
\end{aligned}
$$




$$
\begin{array}{r}
+\frac{\epsilon^{2} n}{h_{s}}\left(-v \cos ^{2} \phi\left(X_{s} Z_{s s}-X_{s s} Z_{s}\right)^{2}+\frac{\partial^{2} v}{\partial s^{2}}-2 \frac{\partial u}{\partial s} \cos \phi\left(X_{s} Z_{s s}-X_{s s} Z_{s}\right)-\right. \\
\left.-u \cos \phi\left(X_{s} Z_{s s s}-X_{s s s} Z_{s}\right)+w \sin \phi \cos \phi\left(X_{s} Z_{s s}-X_{s s} Z_{s}\right)^{2}\right) \\
+\left(1+2 \epsilon n \cos \phi\left(X_{s} Z_{s s}-X_{s s} Z_{s}\right)\right) \frac{\partial v}{\partial n} \\
\left.+n h_{s} \frac{\partial^{2} v}{\partial n^{2}}-\epsilon\left(\frac{\partial v}{\partial \phi}-w\right) \sin \phi\left(X_{s} Z_{s s}-X_{s s} Z_{s}\right)+\frac{h_{s}}{n}\left(\frac{\partial^{2} v}{\partial \phi^{2}}-v-2 \frac{\partial w}{\partial \phi}\right)\right\}(8)
\end{array}
$$

and

$$
\begin{array}{r}
h_{s}\left(\epsilon \frac{\partial w}{\partial t}+\epsilon u \sin \phi\left(Z_{s t} X_{s}-X_{s t} Z_{s}\right)+v \frac{\partial w}{\partial n}+\frac{w}{n} \frac{\partial w}{\partial \phi}+\frac{v w}{n}\right) \\
+\epsilon u \frac{\partial w}{\partial s}+\epsilon \sin \phi\left(X_{s} Z_{s s}-X_{s s} Z_{s}\right) u^{2} \\
=\left(-\frac{1}{n} \frac{\partial p}{\partial \phi}+\frac{2 \epsilon}{R b} u \sin \phi+\frac{\epsilon \sin \phi}{R b^{2}}\left(Z X_{s}-(X+1) Z_{s}-\epsilon n \cos \phi\right)\right) h_{s} \\
+\frac{1}{R e} \frac{1}{\epsilon n}\left\{\frac{-\epsilon^{3} n^{2} \cos \phi\left(X_{s} Z_{s s s}-X_{s s s} Z_{s}\right)}{h_{s}^{2}}\left(\frac{\partial w}{\partial s}+u \sin \phi\left(X_{s} Z_{s s}-X_{s s} Z_{s}\right)\right)\right. \\
+\frac{\epsilon^{2} n}{h_{s}}\left(-w \sin ^{2} \phi\left(X_{s} Z_{s s}-X_{s s} Z_{s}\right)^{2}+\frac{\partial^{2} w}{\partial s^{2}}+2 \frac{\partial u}{\partial s} \sin \phi\left(X_{s} Z_{s s}-X_{s s} Z_{s}\right)\right. \\
\left.+u \sin \phi\left(X_{s} Z_{s s s}-X_{s s s} Z_{s}\right)+v \sin \phi \cos \phi\left(X_{s} Z_{s s}-X_{s s} Z_{s}\right)^{2}\right) \\
+\left(1+2 \epsilon n \cos \phi\left(X_{s} Z_{s s}-X_{s s} Z_{s}\right)\right) \frac{\partial w}{\partial n} \\
\left.+n h_{s} \frac{\partial^{2} w}{\partial n^{2}}-\epsilon\left(\frac{\partial w}{\partial \phi}+v\right) \sin \phi\left(X_{s} Z_{s s}-X_{s s} Z_{s}\right)+\frac{h_{s}}{n}\left(\frac{\partial^{2} w}{\partial \phi^{2}}-w+2 \frac{\partial v}{\partial \phi}\right)\right\} .(9)
\end{array}
$$

The dimensionless boundary conditions (at $n=R(s, \phi, t)$ ) are the kinematic condition

$$
\begin{gathered}
h_{s}\left(\epsilon \frac{\partial R}{\partial t}+\cos \phi\left(X_{t} Z_{s}-X_{s} Z_{t}\right)-v+\frac{\partial R}{\partial \phi} \frac{w}{n}+\frac{\partial R}{\partial \phi} \frac{1}{n} \sin \phi\left(X_{t} Z_{s}-X_{s} Z_{t}\right)\right) \\
+\epsilon u \frac{\partial R}{\partial s}-\epsilon \frac{\partial R}{\partial s}\left(X_{t} X_{s}+Z_{t} Z_{s}+\epsilon n \cos \phi\left(X_{s} Z_{s s}-X_{s s} Z_{s}\right)\right)=0, \quad(10)
\end{gathered}
$$

the tangential stress conditions

$$
\begin{gathered}
\left(1-\epsilon^{2}\left(\frac{\partial R}{\partial s}\right)^{2} \frac{1}{h_{s}^{2}}\right)\left[\epsilon \frac{\partial v}{\partial s}+h_{s} \frac{\partial u}{\partial n}-\epsilon u \cos \phi\left(X_{s} Z_{s s}-X_{s s} Z_{s}\right)\right] \\
+2 \epsilon \frac{\partial R}{\partial s}\left[\frac{\partial v}{\partial n}-\epsilon \frac{\partial u}{\partial s} \frac{1}{h_{s}}-\epsilon \frac{v \cos \phi-w \sin \phi}{h_{s}}\left(X_{s} Z_{s s}-X_{s s} Z_{s}\right)\right]=0,(11)
\end{gathered}
$$




$$
\begin{aligned}
(1 & \left.-\left(\frac{\partial R}{\partial \phi}\right)^{2} \cdot \frac{1}{R^{2}}\right)\left(\frac{\partial w}{\partial n}-\frac{w}{R}+\frac{\partial v}{\partial \phi} \cdot \frac{1}{R}\right) \\
+ & 2 \frac{\partial R}{\partial \phi} \cdot \frac{1}{R}\left(\frac{\partial v}{\partial n}-\frac{1}{R}\left(\frac{\partial w}{\partial \phi}+v\right)\right)=0
\end{aligned}
$$

and the normal stress condition

$$
\begin{gathered}
p-\frac{2}{R e} \frac{1}{E^{2}}\left\{\frac{\epsilon^{2}}{h_{s}^{3}}\left(\frac{\partial R}{\partial s}\right)^{2}\left[\frac{\partial u}{\partial s}+(v \cos \phi-w \sin \phi)\left(X_{s} Z_{s s}-X_{s s} Z_{s}\right)\right]\right. \\
+\frac{1}{\epsilon} \frac{\partial v}{\partial n}+\frac{1}{\epsilon R^{3}}\left(\frac{\partial R}{\partial \phi}\right)^{2}\left(\frac{\partial w}{\partial \phi}+v\right)-\epsilon \frac{\partial R}{\partial s} \frac{1}{h_{s}}\left[\frac{\partial v}{\partial s} \frac{1}{h_{s}}\right. \\
\left.\quad+\frac{1}{\epsilon} \frac{\partial u}{\partial n}-\frac{u}{h_{s}} \cos \phi\left(X_{s} Z_{s s}-X_{s s} Z_{s}\right)\right] \\
-\frac{1}{\epsilon} \frac{\partial R}{\partial \phi} \frac{1}{R}\left(\frac{\partial w}{\partial n}-\frac{w}{R}+\frac{\partial v}{\partial \phi} \cdot \frac{1}{R}\right)+\epsilon \frac{\partial R}{\partial s} \frac{\partial R}{\partial \phi} \frac{1}{h_{s} R}\left[\frac{1}{\epsilon} \frac{\partial u}{\partial \phi} \frac{1}{R}\right. \\
\left.\left.+\frac{u}{h_{s}} \sin \phi\left(X_{s} Z_{s s}-X_{s s} Z_{s}\right)+\frac{\partial u}{\partial s} \frac{1}{h_{s}}\right]\right\}=\frac{\kappa}{W e},
\end{gathered}
$$

where

$$
\begin{gathered}
\kappa=\frac{1}{h_{s}}\left(\epsilon^{2} \frac{\partial}{\partial s}\left(-\frac{1}{h_{s} E} \frac{\partial R}{\partial s}\right)+\frac{1}{n} \frac{\partial}{\partial n}\left(\frac{n h_{s}}{E}\right)+\frac{\partial}{\partial \phi}\left(-\frac{h_{s}}{n^{2} E} \frac{\partial R}{\partial \phi}\right)\right) \\
h_{s}=1+\epsilon n \cos \phi\left(X_{s} Z_{s s}-X_{s s} Z_{s}\right) \\
E=\left(1+\frac{\epsilon^{2}}{h^{2}}\left(\frac{\partial R}{\partial s}\right)^{2}+\frac{1}{n^{2}}\left(\frac{\partial R}{\partial \phi}\right)^{2}\right)^{1 / 2}
\end{gathered}
$$

The arclength condition is

$$
\begin{array}{r}
X_{s}^{2}+Z_{s}^{2}=1 \\
\text { and } \quad v=w=0 \quad \text { on } \quad n=0,
\end{array}
$$

since on the centreline of the jet there must be purely tangential flow. The dimensionless parameters in these equations are the Weber number $W e=\rho U^{2} a / \sigma$, the Rossby number $R b=U /\left(s_{0} \Omega\right)$, the aspect ratio $\epsilon=a / s_{0}$ and the Reynolds number based on radius of the cylinder $R e=\rho U s_{0} / \mu$. 


\section{Asymptotic analysis}

We assume that the jet is a long, slender object and we expand $u, v, w, p$ in Taylor series in $\epsilon n$ (see Eggers [21], Hohman et al. [30]) and $R, X, Z$ in asymptotic series in $\epsilon$ :

$$
\begin{array}{r}
u=u_{0}(s, t)+(\epsilon n) u_{1}(s, \phi, t)+(\epsilon n)^{2} u_{2}(s, \phi, t)+\cdots \\
v=(\epsilon n) v_{1}(s, \phi, t)+(\epsilon n)^{2} v_{2}(s, \phi, t)+\cdots \\
w=(\epsilon n) w_{1}(s, \phi, t)+(\epsilon n)^{2} w_{2}(s, \phi, t)+\cdots \\
p=p_{0}(s, \phi, t)+(\epsilon n) p_{1}(s, \phi, t)+\cdots \\
R=R_{0}(s, t)+\epsilon R_{1}(s, \phi, t)+\cdots \\
X=X_{0}(s)+\epsilon X_{1}(s, t)+\cdots \\
Z=Z_{0}(s)+\epsilon Z_{1}(s, t)+\cdots
\end{array}
$$

We suppose that the radius and the axial component of velocity do not depend on $\phi$ at leading order, as should be expected in a slender jet theory. We further assume that the position of the centreline is not affected by the small perturbations, and at leading order, it is not timedependent. This is as observed in the experiments of Wong et al. [31] when the instability is convective and travels downstream as a growing wave, as assumed here. In fact, in all the experimental observations [31], where the instability was convective, the centreline was always observed to be steady in a frame moving with the rotating container. (However in [31], there was another set of experimental observations, so-called "mode 4" observations, where the instability was an absolute instability, and in that case the centreline was observed to be unsteady.) In section 6 , we consider an unsteady centreline and verify the steady centreline assumption computationally, but for now we assume the centreline of the jet to be steady. From now on, we write $X_{0}$ and $Z_{0}$ as $X$ and $Z$ respectively, for simplicity and we denote $S(s)=X_{s} Z_{s s}-X_{s s} Z_{s}$. We then substitute these expansions into equations (6), (8)-(9), (11)(16) and (18) and, after a few manipulations (see Partridge [32] for details), we obtain that

$$
u_{1}=u_{0} S \cos \phi, \quad u_{2}=\frac{3}{2} u_{0 s} \frac{R_{0 s}}{R_{0}}+\frac{u_{0 s s}}{4} \text { and } v_{1}=-\frac{u_{0 s}}{2} .
$$

A solvability condition is also obtained by requiring $R_{1}$ to be periodic in $\phi$, as in the inviscid case [4], giving

$$
u_{0}^{2} S-\frac{2}{R b} u_{0}+\frac{(X+1) Z_{s}-Z X_{s}}{R b^{2}}-\frac{3}{R e} u_{0 s} S-\frac{S}{W e R_{0}}=0 .
$$


Is is worth noting that the difference in the solvability condition between the inviscid case and the viscous case is given by the term $\frac{3}{R e} u_{0 s} S$.

The first Navier-Stokes equation (7) at order $\epsilon$ can be written as

$$
u_{0 t}+u_{0} u_{0 s}=-\frac{1}{W e}\left(\frac{1}{R_{0}}\right)_{s}+\frac{(X+1) X+Z Z_{s}}{R b^{2}}+\frac{3}{R e} \frac{\left(R_{0}^{2} u_{0 s}\right)_{s}}{R_{0}^{2}}
$$

From the kinematic condition (10), we obtain at leading order $\epsilon$ the equation

$$
R_{0 t}+\frac{u_{0 s}}{2} R_{0}+u_{0} R_{0 s}=0 .
$$

The last equation to be solved is the arclength equation (17) at leading order

$$
X_{s}^{2}+Z_{s}^{2}=1 .
$$

So we obtained the equations (20)-(23) for the unknowns $u_{0}, R_{0}, X$ and $Z$. A comparison between these equations and the ones for a viscous bending jet, derived using a different method, by Entov and Yarin [26] and Yarin [27] is presented in the Appendix.

\section{Steady state solutions}

First we will search for steady-state solutions of the previous equations. Now we consider all the variables to be only functions of $s$. The equations in the steady case are

$$
\begin{array}{r}
u_{0} u_{0 s}=-\frac{1}{W e}\left(\frac{1}{R_{0}}\right)_{s}+\frac{(X+1) X+Z Z_{s}}{R b^{2}}+\frac{3}{R e} \cdot \frac{\left(R_{0}^{2} u_{0 s}\right)_{s}}{R_{0}^{2}}, \\
\frac{u_{0 s}}{2} R_{0}+u_{0} R_{0 s}=0 \\
\left(X_{s} Z_{s s}-X_{s s} Z_{s}\right)\left(u_{0}^{2}-\frac{3}{R e} u_{0 s}-\frac{1}{W e R_{0}}\right) \\
-\frac{2}{R b} u_{0}+\frac{(X+1) Z_{s}-Z X_{s}}{R b^{2}}=0 \\
X_{s}^{2}+Z_{s}^{2}=1 .
\end{array}
$$

From equation (25) we observe that $R_{0}^{2} u_{0}$ is constant and, by using $R_{0}(0)=1$ and $u_{0}(0)=1$, we have

$$
R_{0}^{2} u_{0}=1
$$


so we can substitute $R_{0}$ in the previous equations, which now become

$$
\begin{array}{r}
u_{0} u_{0 s}=-\frac{1}{W e} \frac{u_{0 s}}{2 \sqrt{u_{0}}}+\frac{(X+1) X+Z Z_{s}}{R b^{2}}+\frac{3}{R e}\left(u_{0 s s}-\frac{u_{0 s}^{2}}{u_{0}}\right) \\
\left(X_{s} Z_{s s}-X_{s s} Z_{s}\right)\left(u_{0}^{2}-\frac{3}{R e} u_{0 s}-\frac{\sqrt{u_{0}}}{W e}\right) \\
-\frac{2}{R b} u_{0}+\frac{(X+1) Z_{s}-Z X_{s}}{R b^{2}}=0, \\
X_{s}^{2}+Z_{s}^{2}=1 .
\end{array}
$$

The unknown for the equations (29)-(31) are $X, Z, u_{0}$. For the inviscid case we were able to use a Runge-Kutta method to calculate the steady states numerically. In the viscous case the method does not work, so we built a different scheme which uses a second order centred finite difference discretization of the equations.

The boundary conditions at the nozzle are $X(0)=Z(0)=Z_{s}(0)=$ $0, u_{0}(0)=X_{s}(0)=1$. The unknowns used in the scheme were in fact $X_{s}, Z_{s}$ and $u_{0}$ and the values of $X$ and $Z$ are obtained by trapezoidalrule integration. The downstream boundary conditions are obtained by quadratic extrapolation of the last internal mesh points. The initial guess is taken as a straight cylinder of constant radius, without rotation, and then we put small amounts of rotation and viscosity until we obtain the desired values of parameters $R b$ and $R e$. The nonlinear equations are solved at each step using Newton's method or a modified Newton's method (the Jacobian is computed only for the first iteration). The guess at next step uses the previously calculated solution. We usually obtain in only a few iterations solutions for very viscous flows, which rotate very fast. A similar method was used by Hohman et al. [30] in a different context, to solve the equations for a electrically forced jet (see also Reneker et al. [33], Yarin et al. [34] for a derivation of the quasi-one dimensional equations for this case). The numerical accuracy was checked by varying the number of mesh points $M$ and the grid interval $d s$. Usually, the results were found to be independent of $M$ and $d s$, within graphical accuracy, for $M \geq 200$ and $d s \leq 0.1$.

The solutions in the inviscid case were compared with the solutions obtained using a Runge-Kutta method, as in Wallwork et al. [1], and a very good agreement was found (see Fig. 1).

Figure 2 shows the centreline of the steady jet for various Reynolds numbers, the other parameters being fixed. As viscosity increases, the centreline becomes more tightly coiled, but only for small Reynolds numbers the deviation from the inviscid centreline is significant. It is worth noting that in the experiments the range of Reynolds number 
was between 100 and 300,000. The effect of viscosity is to slow the decrease of steady jet radius with arclength, along the jet.

The influence of the Weber number and Rossby number for a viscous jet, shown in Fig. 3, is the same as in the inviscid case: the centreline becomes more tightly coiled when the Weber number decreases (the surface tension increases) or when the Rossby number decreases (the rotation rate increases).

It was previously found (see Wallwork et al. [1]) that there is a singularity at $W e=1$. We present two numerical solutions for the Weber number close to one, in the inviscid case in Fig. 4. The radius seems to be a periodic function of the arclength, as in the case of the annular liquid membranes subject to gravity where similar solutions were found (see Ramos [35], Fig. 2). The centreline has the form of a self-intersecting curve. For $W e>1$ the jet tends to go in the clockwise direction and for $W e<1$ the jet will move anti-clockwise (the container rotates anti-clockwise). Both solutions have no physical significance, which was also mentioned by Ramos [35] for his solutions for annular liquid membranes.

\section{Nonlinear temporal solutions}

The nonlinear system to be solved is (21)-(22). We replace the leading order pressure $p_{0}=\frac{1}{W e} \frac{1}{R_{0}}$ by the expression for the full curvature which contains only $R_{0}$ and is not $\phi$-dependent, namely

$$
p=\frac{1}{W e}\left[\frac{1}{R_{0}\left(1+\epsilon^{2} R_{0_{s}}^{2}\right)^{1 / 2}}-\frac{\epsilon^{2} R_{0_{s s}}}{\left(1+\epsilon^{2} R_{0_{s}}^{2}\right)^{3 / 2}}\right] .
$$

This method, abandoning the formal asymptotic analysis on physical grounds, was applied with good results by many authors to prevent instability to wave modes with zero wavelength (see for example Lee [10], and, latter, Papageorgiou and Orellana [13], Eggers \& Duppont [18]). Including this full expression for the curvature, the linear instability of this model gives results which agree with experiments; without this expression for the full curvature the linear instability results do not agree with experimental instability results even for a straight jet. It is also worth noting that Entov and Yarin [26, p.46] and Yarin [27] have obtained the pressure in the form (32) in their derivation of the quasi-one-dimensional equations for bending jets, using physical arguments.

The main difference between our equations and the reduced axisymmetric jet is the term containing $R b$. 
If we denote $A=R_{0}^{2}$ and $u=u_{0}$ the system (21)-(22) can be rewritten as

$$
\begin{gathered}
A_{t}+(A u)_{s}=0 \\
u_{t}+\left(\frac{u^{2}}{2}\right)_{s}=-\frac{1}{W e} \frac{\partial}{\partial s} \frac{4\left(2 A+\left(\epsilon A_{s}\right)^{2}-\epsilon^{2} A A_{s s}\right)}{\left(4 A+\left(\epsilon A_{s}\right)^{2}\right)^{3 / 2}} \\
+\frac{(X+1) X_{s}+Z Z_{s}}{R b^{2}}+\frac{3}{R e} \cdot \frac{\left(A u_{s}\right)_{s}}{A}
\end{gathered}
$$

We solve the system (33)-(34) for $s \in[0, l]$ where $l$ is the length of the (numerical) jet. A suitable numerical method can be obtained by modifying the method of Zhu et al. [36], which was used to solve numerically a liquid jet falling into a liquid pool. This system is solved using a finite-difference method. The spatial grid is fixed and uniform, as we define $M$ equally spaced points $s_{0}=0$ (the nozzle), $s_{i}=s_{0}+i d s$, $i=1, M$ where $d s$ is the spatial grid interval. The magnitude of the time step is denoted by $d t$. The time integration method is based on a explicit scheme, using the Lax-Wendroff method two-stage scheme (see e.g. $[37$, p.835]).

The initial conditions at $t=0$ were given by the steady solutions calculated using the method described in the previous section

$$
A(s, 0)=R_{0}^{2}(s), \quad u(s, 0)=u_{0}(s) .
$$

The value of $\epsilon=a / s_{0}$ can be measured from experiments. In the calculations we used $\epsilon=0.01$ which is typical in experiments and in industrial problems [2],[31]. For a straight uniform jet $A(s, 0)=$ $1, u(s, 0)=1$.

We impose the upstream boundary conditions at the nozzle

$$
A(0, t)=1, \quad u(0, t)=1+\delta \sin \left(K \frac{t}{\epsilon}\right) .
$$

We should note also that the non-dimensional wave numbers are equivalent to disturbance frequency, so $K$ (which is the disturbance frequency) fixes the wavelength of the perturbation. We can also change the initial conditions, to make an initial perturbation of radius, rather than of velocity.

The downstream boundary conditions are obtained by quadratic extrapolation of the last internal mesh points. The specification of (numerical) boundary conditions at the nozzle is not simple. Sirignano and Mehring [38] present a more detailed discussion for annular sheets, which is close to our problem. The boundary conditions at the far end of the liquid jet have an influence only in a narrow region close to it, except 
for small Weber numbers (see Yarin [27]). For small Weber number an absolute instability can develop on the jet and the numerical simulation becomes rather difficult. Similar problems were found by Mehring and Sirignano [39] for annular or conical liquid sheets. Earlier work on the absolute instability in the context of Cosserat fluid jet equations can be found in Bogy et al. [40]. The question of the absolute instability and its relation to the dripping/jet transition for small Weber numbers (close to 3 or less) is discussed in detail by Lin and Reitz [41].

In the simulations we choose that the jet-breakup occurs when the minimum dimensionless radius of the jet is less then a small value, arbitrarily chosen (usually $5 \%$ of the initial radius). Downstream of breakup point, the jet solution no longer has physical meaning, since the jet in that region will have broken up into droplets, which cannot be described by this approach, as is also the case in other works.

The accuracy of the numerical simulations is checked by refining the mesh and changing the time-step. An example is presented in Fig. 5 .

The breakup length against a dimensionless viscosity scale (namely the Ohnesorge number $O h=\mu / \sqrt{\sigma a \rho}$ ) is plotted in Fig. 6 and, as for the axisymmetric case, it can be observed that it increases with viscosity. The breakup length against the Rossby number is also plotted and it is observed that it increases with the rotation rate. The breakup length is dimensionless, after being divided by the initial radius $a$.

The jet profile and the radius for two different Reynolds numbers are presented in Fig. 7. The trajectory is almost the same, as the viscosity does not have a big influence on it, but the breakup length is quite different. It can be observed how the viscosity diminishes the growth factor of the disturbance at the most unstable wavenumber and so the breakup is delayed. The most unstable wave number also decreases with the viscosity. For example, when the magnitude of disturbance $\delta=0.1$, for the inviscid jet the most unstable wavenumber is approximately 0.94 , while for a viscous jet $(R e=1000)$ it is approximately 0.835 and for a more viscous jet $(R e=600)$ the most unstable wavenumber is approximately 0.795 .

The jet profile and the radius for two different Rossby numbers are presented in Fig. 8. We observe that, down the jet, the wavelength of the perturbation increases with the rotation rate and the growth factor of the disturbance diminishes.

In Fig. 9-11 we compare the drop sizes predicted by the model, for a fixed oscillation magnitude, when the frequency $K$ is varied from 0 to 1 . The main drop and the satellite drop volume were obtained by numerical integration of the jet profile at the breakup time, using the trapezoidal rule. The satellite drop was defined by two minima of 
the radius, when they exist. Where there are more satellite droplets, we consider that all satellite droplets merge into one satellite droplet. The satellite and the main drop were supposed to become spheres after jet rupture and their radius is plotted in these graphs. In Fig. 9(a) all the parameters were kept constant, except for the Rossby number. We observe that the satellite droplet radius increases with increasing rotation rate (i.e. decreasing $R b$ ) and the main drop radius decreases with increasing rotation rate. It is worth noting that this behaviour has been observed in experiments (see Wong et al. [31] and Partridge et al. [42]). In particular, we reproduce Figure 9 from Partridge et al. [42] here as Figure 9(b). The graph shows an experimental drop size distribution diagram. The shape of the distribution can be seen to be bi-modal, with the satellites shown on the left of each bi-modal distribution curve. It can be seen that the qualitative behaviour in Figure 9(b) is the same as in our theoretical results shown in Figure 9(a) with regard to how the sizes of the main and satellite drops vary with $R b$ (i.e. compare how the location of the two maxima in the bimodal distribution vary with rotation rate in 9 (b) with the theoretical results). Note, in experiments it is not possible to fix the wavenumber $k$, and a random distribution of a set of $k$ 's located about the most unstable wavenumber occurs creating a distribution. Because of this, we are currently extending the experiments of Partridge et al. [42] by exciting a single particular value of $k$ using acoustic insonification via a loudspeaker located inside the container. Preliminary experimental results are shown in [32], and further experiments will be reported at a latter date. This will lead to a more detailed comparison between theory and experiments.

In Fig. 10 the Reynolds number is varied and we obtained that the main drop radius increases with the viscosity and the satellite droplet radius decreases. This conclusion was also true for the (temporal) instability analysis for an axisymmetric jet, given by Ashgriz and Mashayek [43]. For small values of $k$ the 'satellite' droplet radius can be sometimes bigger than the 'main' drop radius. In Fig. 11 the influence of the Weber number is studied. In this case, the satellite droplet radius seems to increase with the Weber number and the main drop radius decreases; but the influence of the Weber number is not as important as that of the other parameters.

The effect of the disturbance amplitude $\delta$ on main droplet and satellite droplet radius is investigated in Fig. 12 for two different sets of parameters. We observed that the main droplet radius and the satellite droplet radius varies slowly when the disturbance amplitude increases, for a fixed disturbance frequency. It can be observed that for $\delta$ greater than some $\delta_{0}$, which depends on the parameters of the problem, the main droplet radius decreases and the satellite droplet radius increases 
when the disturbance amplitude increases. A similar behaviour was obtained by Hilbing and Heister [19] (see their Fig. 5) for axisymmetric finite-length jets, who used a boundary element method to solve the full nonlinear problem. This behaviour reverses for $\delta$ smaller than $\delta_{0}$.

We also compared the breakup length obtained in numerical simulations with ones obtained in a set of experiments [31]. In these experiments, the radius of the orifice $a$ is $0.5 \mathrm{~mm}$, the radius of the container $s_{0}$ is $145 \mathrm{~mm}$, and the viscosity of the liquid $\approx 4 \mathrm{mPas}$. The container was rotated at 4 differents rates, from 50 to 250 rotations per minute. To compare the numerical simulation with the experiments, we fixed $K$ to be the most unstable frequency in all cases (i.e. the one which gives the shortest jets in each case) and we fixed the magnitude of the disturbance $\delta$ such that the theoretical and experimental breakup length in one of the cases was identical. This gave the fitting parameter $\delta=0.0001$. The results, shown in Fig. 13, are encouraging, with the numerical simulations capturing quite well the experimental results.

\section{Asymptotic analysis when the centreline is time-dependent}

We now consider the leading order components $X$ and $Z$ of the centreline coordinates to be time-dependent and denote $E=X_{t} Z_{s}-X_{s} Z_{t}$. To balance the kinematic condition at the leading order, we should consider leading order terms in the expansions of $v$ and $w$. They are now

$$
v=v_{0}(s, \phi, t)+\epsilon n v_{1}+\ldots, w=w_{0}(s, \phi, t)+\epsilon n w_{1}+\ldots,
$$

with the other expansions being unchanged from (19).

From the kinematic condition at leading order and the conservation of mass equation we obtain

$$
v_{0}(s, \phi, t)=E \cos \phi \text { and } w_{0}(s, \phi, t)=-E \sin \phi .
$$

Following the previous asymptotic analysis, we will obtain, by considering the second tangential stress condition

$$
v_{1 \phi}=0, w_{1 \phi}=0 .
$$

From the continuity equation and from the first tangential stress condition we find

$$
v_{1}=-\frac{u_{0 s}}{2}-\frac{S E}{2}, \quad u_{1}=\left(u_{0} S-E_{s}\right) \cos \phi, \text { and }
$$




$$
u_{2}=\frac{3}{2}\left(u_{0 s}+S E\right) \frac{R_{0 s}}{R_{0}}+\frac{u_{0 s s}+(S E)_{s}}{4} .
$$

From the normal stress condition we obtain the new solvability condition, by requiring that $R_{1}$ is periodic in $\phi$, namely

$$
\begin{aligned}
E_{t}+ & u_{0}\left(X_{s t} Z_{s}-Z_{s t} X_{s}\right)+u_{0} E_{s}-\frac{E}{2}\left(u_{0 s}+S E\right)+\frac{3}{R e} E S^{2}= \\
& =u_{0}^{2} S-\frac{2}{R b} u_{0}+\frac{(X+1) Z_{s}-Z X_{s}}{R b^{2}}-\frac{3}{R e} u_{0 s} S-\frac{S}{W e R_{0}},
\end{aligned}
$$

and $w_{1} E=0$. The right-hand side of this equation is exactly equation (20). In the second equation, we can take $w_{1}=0$. The first NavierStokes equation (7) at order $\epsilon$ becomes

$$
\begin{gathered}
u_{0 t}+E\left(Z_{s t} X_{s}-X_{s t} Z_{s}\right)+u_{0} u_{0 s}+2 u_{0} E S-E E_{s}= \\
-\frac{1}{W e}\left(\frac{1}{R_{0}}\right)_{s}+\frac{2}{R b} E+\frac{(X+1) X_{s}+Z Z_{s}}{R b^{2}}+\frac{3}{R e} \frac{\left(R_{0}^{2}\left(u_{0 s}+S E\right)\right)_{s}}{R_{0}^{2}}(36)
\end{gathered}
$$

The kinematic condition (10)at order $\epsilon$ is

$$
R_{0 t}+\frac{u_{0 s}}{2} R_{0}+\frac{S}{2} E R_{0}+u_{0} R_{0 s}-R_{0 s}\left(X_{t} X_{s}+Z_{t} Z_{s}\right)=0,
$$

or, using $\left(u_{0}-X_{t} X_{s}-Z_{t} Z_{s}\right)_{s}=u_{0 s}+S E$, which can be obtained after a few calculations, we can put it in the equivalent form

$$
R_{0 t}+\left(u_{0}-X_{t} X_{s}-Z_{t} Z_{s}\right)_{s} \frac{R_{0}}{2}+R_{0 s}\left(u_{0}-X_{t} X_{s}-Z_{t} Z_{s}\right)=0 .
$$

The equations (35)-(37) and the arclength condition (23) are the new equations for the unknowns $u_{0}, R_{0}, X$ and $Z$.

We do not solve these full equations, but we consider some small perturbations of the steady centreline from section 4 by writing $X(s, t)=$ $X_{0}(s)+\hat{X}(s, t)$ and $Z(s, t)=Z_{0}(s)+\hat{Z}(s, t)$ and then linearize in $\hat{X}$ and $\hat{Z}$. Figure 14 shows the perturbation of the trajectory of the jet from its initial conditions plotted against $s$ for two times $t$ up to the time of break-up. The maximum deviation is of order $10^{-2}$ and is relatively small compared to the $O(1)$ values of $X_{0}(s)$ and $Z_{0}(s)$ (e.g. see figures 1 to 4$)$ : hence the trajectory is effectively steady. The maximum deviation increases with viscosity (see Fig. 15). 


\section{Conclusions}

The evolution of a disturbance on a rotating, slender viscous jet emanating from a small orifice up to the breakup point has been studied. The nonlinear partial differential equations which describe this evolution were obtained by using an asymptotic approach.

A numerical method based on finite differences and the Newton method was developed to solve the nonlinear differential equations for the steady-state solutions. The viscosity was shown to have a small influence on the trajectory of the centreline and on the steady radius of the jet, except for the very viscous fluids when it becomes more important.

The numerical method used to solve the time-dependent system was described and the influence of various parameters on the solutions have been quantified. It was found that the breakup length increases with viscosity and with the rotation rate. The satellite and the main drop radius were also computed and a good agreement was found in some cases with the experimental results of Partridge et al. [42]. The experiments carried out by Partridge et al. [42] are currently being extended by using acoustic insonification via a loudspeaker to control the most unstable wavenumber and will be compared in the future with the analytical model.

When the centreline is assumed to be time-dependent, a new system of nonlinear partial differential equations is derived, but the deviations from the steady trajectory are found to be small, except for very viscous jets (see the experimental results [31]).

The methods developed here can be applied for other physical problems. Uddin et al. [44], who studied the linear instability of rotating power law non-Newtonian liquid jets, is currently extending this nonlinear model to his case. Also, the full three-dimensional problem with both gravity and rotation can be studied in the same manner.

\section{Appendix}

We compare in this Appendix the quasi-one-dimensional equations derived by Entov \& Yarin [26] for a bending jet with the equations obtained in our asymptotic derivation. Their quasi-one dimensional equations, when the jet axis is a curve lying in a plane [Entov \& Yarin $[26$, p.101] eq (4.17)], are

$$
\frac{\partial \lambda f}{\partial t}+\frac{\partial f W}{\partial \breve{s}}=0
$$




$$
\begin{aligned}
& \frac{\partial \lambda f V_{\tau}}{\partial t}-\frac{f V_{n}}{\lambda} \frac{\partial \lambda V_{n}}{\partial \breve{s}}+\frac{\partial f V_{\tau} W}{\partial \breve{s}}-\lambda f W k V_{n}=\frac{1}{\rho} \frac{\partial P}{\partial \breve{s}}+\lambda f F_{\tau}+\frac{1}{\rho} \lambda q_{\tau},(39) \\
& \frac{\partial \lambda f V_{n}}{\partial t}+\frac{f V_{\tau}}{\lambda} \frac{\partial \lambda V_{n}}{\partial \breve{s}}+\frac{\partial f V_{n} W}{\partial \breve{s}}+\lambda f W k V_{\tau}=\frac{1}{\rho} \lambda k P+\lambda f F_{n}+\frac{1}{\rho} \lambda q_{n}, \quad(40) \\
& \lambda=\left[1+\left(\frac{\partial H}{\partial \breve{s}}\right)^{2}\right]^{1 / 2}, \quad k=\frac{\partial^{2} H}{\partial s^{2}} \lambda^{-3}, \quad \frac{\partial H}{\partial t}=\lambda V_{n}, \quad W=V_{\tau}-V_{n} \frac{\partial H}{\partial \breve{s}} .
\end{aligned}
$$

Here $f=\pi R^{2}(\breve{s}, t)$ is the area of the jet cross-section and $F_{\tau}, F_{n}$ are the components of the external force $F$ per unit mass. The resultant action of the external loads is specified by the linear density of forces $q$ applied to the jet axis and is taken to be zero.

Assuming the jet motion to be such that the tangent to the jet axis at any instant and at all points makes an acute angle with a straight line $O_{1} \xi$, the jet axis in their case is described in the Cartesian coordinate by

$$
\mathbf{R}=\mathbf{i} \breve{s}+\mathbf{k} H(\breve{s}, t),
$$

while our jet axis, which does not have to satisfy such a restriction, is

$$
\mathbf{R}=\mathbf{i} X(s, t)+\mathbf{k} Z(s, t) .
$$

The relations between the coordinates systems is

$$
\breve{s}=X(s, t), H(\breve{s}, t)=Z(s, t),
$$

and it follows $\frac{\partial H}{\partial \breve{s}}=\frac{Z_{s}}{X_{s}}, \lambda=\frac{1}{X_{s}}, k=Z_{s s} X_{s}-X_{s s} Z_{s}(=S), \frac{\partial H}{\partial t}=$ $Z_{t}-\frac{Z_{s}}{X_{s}} X_{t}=-\frac{E}{X_{s}}$. Generally, for a function $\breve{a}(\breve{s}, t)=\breve{a}(X(s, t), t):=$ $a(s, t)$, we have $\frac{\partial \breve{a}}{\partial \breve{s}}=\frac{1}{X_{s}} \frac{\partial a}{\partial s}, \frac{\partial \breve{a}}{\partial t}=\frac{\partial a}{\partial t}-\frac{X_{t}}{X_{s}} \frac{\partial a}{\partial s}$.

The velocities $V_{\tau}$ and $V_{n}$ are the components of the velocity fields on the centreline, in tangential and normal direction

$$
\tau=\mathbf{e}_{\mathbf{s}}=X_{s} \mathbf{i}+Z_{s} \mathbf{k} \quad \text { and } n=-Z_{s} \mathbf{i}+X_{s} \mathbf{k},
$$

and are found to be $V_{\tau}=u, V_{n}=-E$. The longitudinal force $P$ on the cross-section becomes in the inviscid case (see Entov\& Yarin [26], eq. (4.16), after a few manipulations)

$$
P=\sigma \pi R^{2}\left(\frac{1}{R\left(1+R_{s}^{2}\right)^{1 / 2}}+\frac{R_{s s}}{\left(1+R_{s}^{2}\right)^{3 / 2}}\right)+3 \mu \pi R^{2}\left(u_{s}+S E\right) .
$$

The equations (38)-(40) can be rewritten as

$$
\frac{\partial}{\partial t}\left(\frac{R^{2}}{X_{s}}\right)-\frac{X_{t}}{X_{s}} \frac{\partial}{\partial t}\left(\frac{R^{2}}{X_{s}}\right)+\frac{1}{X_{s}} \frac{\partial}{\partial s}\left(R^{2}\left(u+E \frac{Z_{s}}{X_{s}}\right)\right)=0
$$




$$
\begin{gathered}
\frac{\partial}{\partial t}\left(\frac{R^{2} u}{X_{s}}\right)-\frac{X_{t}}{X_{s}} \frac{\partial}{\partial s}\left(\frac{R^{2} u}{X_{s}}\right)-R^{2} E \frac{\partial}{\partial s}\left(\frac{E}{X_{s}}\right)+\frac{1}{X_{s}} \frac{\partial}{\partial s}\left(R^{2} u\left(u+E \frac{Z_{s}}{X_{s}}\right)\right) \\
+\frac{1}{X_{s}} R^{2}\left(u+E \frac{Z_{s}}{X_{s}}\right) S E=\frac{1}{\rho} \frac{\partial}{\partial s}\left(\frac{P}{\pi X_{s}}\right)+\frac{R^{2}}{X_{s}} F_{\tau} \\
\frac{\partial}{\partial t}\left(\frac{R^{2} E}{X_{s}}\right)-\frac{X_{t}}{X_{s}} \frac{\partial}{\partial s}\left(\frac{R^{2} E}{X_{s}}\right)+R^{2} u \frac{\partial}{\partial s}\left(\frac{E}{X_{s}}\right)+\frac{1}{X_{s}} \frac{\partial}{\partial s}\left(R^{2} E\left(u+E \frac{Z_{s}}{X_{s}}\right)\right) \\
-\frac{1}{X_{s}} R^{2} S u\left(u+E \frac{Z_{s}}{X_{s}}\right)=\frac{1}{\rho} S \frac{P}{\pi X_{s}}+\frac{R^{2}}{X_{s}} F_{n} .
\end{gathered}
$$

After nondimensionalizing the equations, and using our asymptotic expansion, at leading order $R$ can be replaced by $R_{0}$ and $u$ by $u_{0}$. Assuming that the centreline is not time-dependent at leading order, it can be observed that equation (41) is exactly equation (22) multiplied by $2 R_{0} / X_{s}$. Again, by multiplying equation (21) with $R_{0}^{2} / X_{s}$ and subtracting equation (41), we will obtain equation (42), with the mention that the term containing $R b$ is included in $F_{\tau}$ and on the right hand side the leading order term $-\frac{1}{W e R_{0}}$ s will appear instead of the pressure term $P_{s}$ containing the full curvature, which is a common feature for any asymptotic analysis with surface tension (see Eggers [21]). The solvability condition (20) can be recovered from the equation (43), with the same observation as above, with the term $-\frac{\left(X_{s} Z_{s s}-X_{s s} Z_{s}\right)}{W e R_{0}}$ instead of $\frac{\left(X_{s} Z_{s s}-X_{s s} Z_{s}\right)}{W e} P$ corresponding to full curvature.

A comparison between the above equations and our equations obtained when the centreline is time-dependent (section 6) can also be performed, with similar conclusions.

\section{References}

1. I.M. Wallwork, S.P. Decent, A.C. King, R.M.S.M. Schulkes, The trajectory and stability of a spiralling liquid jet. Part 1. Inviscid theory. J. Fluid Mech. 459 (2002) 43-65.

2. K.G. Andersen and.G. Yttri, Et fors $\phi k$ verdt - Forskning og utvikling i Norsk Hydro gjennom 90 ar, Universitetsforlaget, Oslo (1997).

3. S.P. Decent, A.C. King and I.M., Wallwork, Free jets spun from a prilling tower. Journal of Engineering Mathematics. 42 (2002) 265-282.

4. E.I. Părău, S.P. Decent, A.C. King, M.J.H. Simmons, D.C.Y. Wong, Nonlinear travelling waves on a spiralling liquid jet. Wave Motion 43 (2006) 599-618.

5. I.M. Wallwork, The trajectory and stability of a spiralling liquid jet. Ph.D. Thesis, Univ. of Birmingham (2002) 259 pp. 
6. S.P. Decent, A.C. King, M.J.H. Simmons, E.I. Părău, D.C.Y. Wong, I.M. Wallwork, The trajectory and stability of a spiralling liquid jet: part II. Viscous Theory. University of Birmingham preprint series (2005) 32 pp.

7. Lord Rayleigh, On the instability of jets. Proc. London Math. Soc. 10 (1878) $4-13$.

8. Lord Rayleigh, 1892. On the instability of a cylinder of viscous liquid under capillary force. Phi. Mag. 34 (1892) 145-154.

9. C. Weber, Zum Zerfall eines Flussigkeitsstrahles. Z. Angew. Math. Mech. 11 (1931) 136-41.

10. H. C. Lee, Drop formation in a liquid jet, IBM J. Res. Dev. 18 (1974) 364-369.

11. N.N. Mansour, T.S. Lundgren, Satellite formation in capillary jet break-up. Phys. Fluids A 2 (1990) 1141-1144.

12. R.M.S.M. Schulkes, Dynamics of liquid jets revised. J. Fluid Mech. 250 (1993) 635-650.

13. D.T. Papageorgiou, O. Orellana, Study of cylindrical jet breakup using onedimensional models of the Euler equations. SIAM J. Appl. Math. 59, 1 (1998) $286-317$.

14. J.B. Keller, S.I. Rubinow and Y.O. Tu, Spatial instability of a jet. Phys. Fluids 16 (1973) 2052-2055.

15. W.T. Pimbley, H.C. Lee, Satellite drop formation in a liquid jet. IBM J. Res. Dev. 21 (1977) 21-30.

16. D.B. Bogy, Use of one-dimensional Cosserat theory to study instability in a viscous liquid jet. Phys. Fluids 21 (1978) 190-197.

17. D.B. Bogy, Wave-propagation and instability in circular semi-infinite liquid jet harmonically forced at nozzle. J. Appl. Mech. 45 (1978) 469-474.

18. J. Eggers and T.F. Dupont, Drop formation in a one-dimensional approximation of the Navier-Stokes equation. J. Fluid Mech. 246 (1994) 205-221.

19. J.H. Hilbing and D.S. Heister, Droplet size control in liquid jet breakup. Phys. Fluids 8, 6 (1996) 1574-1581.

20. B.S. Cheong and T. Howes, Capillary jet instability under the influence of gravity. Chem. Eng. Sci. 59 (2004) 2145-2157.

21. J. Eggers, Nonlinear dynamics and breakup of free-surface flows. Rev. Mod. Phy. 69, 3 (1997) 865-929.

22. J.-M. Vanden-Broeck and J.B. Keller, Jet rising and falling under gravity. $J$. Fluid Mech. 124 (1982) 335-345.

23. F. Dias and J.-M. Vanden-Broeck, Flows emerging from a nozzle and falling under gravity. J. Fluid Mech. 213 (1990) 465-477.

24. D.S. Finnicum, S.J. Weinstein and K.J. Ruschak, The effect of applied pressure on the shape of a two-dimensional liquid curtain falling under the influence of gravity. J. Fluid Mech. 255 (1993) 645-665.

25. L.J. Cummings, P.D. Howell, On the evolution of non-axisymmetric viscous fibres with surface tension, inertia and gravity. J. Fluid Mech. 389 (1999) 361389.

26. V.M. Entov and Y.L. Yarin, The dynamics of thin liquid jets in air. J. Fluid Mech. 140 (1984) 91-111.

27. A.L. Yarin, Free Liquid Jets and Films: Hydrodynamics and Rheology. Longman, New York (1993) 446 pp.

28. G.K. Batchelor An introduction to Fluid Dynamics, Cambridge University Press (1967) 635 pp.

29. F.J. Garcia and A. Castellanos, One-dimensional models for slender axisymmetric viscous liquid jets. Phys. Fluids 6 (1994) 2676-2689. 
30. M.M. Hohman, M. Shin, G. Rutledge and M.P. Brenner, Electrospinning and electrically forced jets. II. Applications. Phys. Fluids 13, 8 (2001) 2221-2236.

31. D.C.Y. Wong, M.J.H. Simmons, S.P. Decent, E.I. Părău, A.C. King, Break-up dynamics and drop size distribution created from curved liquid jets. Int. J. Multiphase Flow 30 (2004) 499-520.

32. L. Partridge, Experimental and theoretical analysis of prilling, Ph. D. Thesis, Univ. of Birmingham (2006).

33. D.H. Reneker, A.L. Yarin, H. Fong, S. Koombhongse, Bending instability of electrically charged liquid jets of polymer solutions in electrospinning. J. Appl. Phys. 87 (2000) 4531-4547.

34. A.L. Yarin, S. Koombhongse, D.H. Reneker, Bending instability in electrospinning of nanofibers. J. Appl. Phys. 89 (2001) 3018-3026.

35. J.I. Ramos, Analysis of Annular liquid membranes and their singularities. Meccanica 32 (1997) 279-293.

36. Y. Zhu, H.N. Oğuz and A. Prosperetti, On the mechanism of air entrainemnt by liquid jets at a free surface. J. Fluid Mech. 404 (2000) 151-177.

37. W. H. Press, S.A. Teukolsky, W.T. Vetterling, B.P. Flannery, Numerical Recipes in Fortran 77: The Art of Scientific Computing; (Fortran Numerical Recipes, Vol 1) (Cambridge University Press, 2001).

38. W.A. Sirignano and C. Mehring Review of theory of distortion and disintegration of liquid streams. Progress in Energy and Combustion Science 26 (2000) 609-655.

39. C. Mehring and W.A. Sirignano, Nonlinear capillary waves on swirling, axisymmetric free liquid films. Int. J. Multiphase Flow 27 (2001) 1701-1734.

40. D.B. Bogy, S.J. Shine, F.E. Talke, Finite difference solution of the Cosserat fluid jet equations. J. Comput. Phys 38 (1980) 249-326.

41. S.P. Lin and R.D. Reitz, Drop and spray formation from a liquid jet. Annu. Rev. Flu. Mech. 30 (1998) 85-105.

42. L. Partridge, D.C.Y. Wong, M.J.H. Simmons, E.I. Părău, S.P. Decent Experimental and theoretical description of the break-up of curved liquid jets in the prilling process. Chem. Eng. Res. Des. 83(A11), 1267-1275 (2005).

43. N. Ashgriz and F. Mashayek, Temporal analysis of capillary gravity jet breakup. J. Fluid Mech. 291 (1995) 163-190.

44. J. Uddin, S.P. Decent, M.J.H. Simmons, The instability of shear thinning and shear thickening spiralling liquid jets: Linear theory, ASME J. Fluid Eng. 128 (2006) 968-975. 

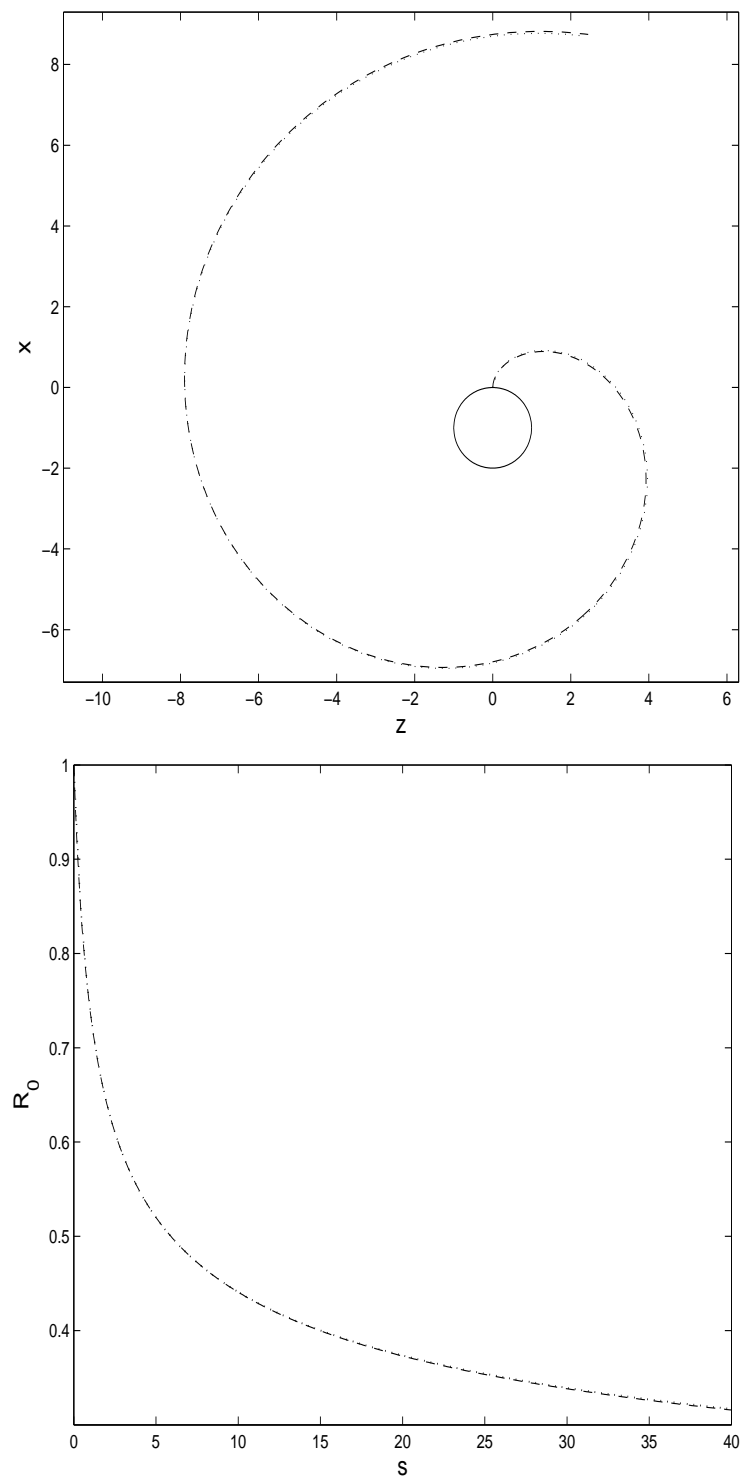

Figure 1. The steady centreline $(z, x)$ and the steady radius $R_{0}$ vs. $s$ in the inviscid case obtained with the Newton method (dotted line) and with the Runge-Kutta method (dashed line). The parameters are for this case $W e=20, R b=1$, $M=400, d s=0.1, R e=\infty$. 

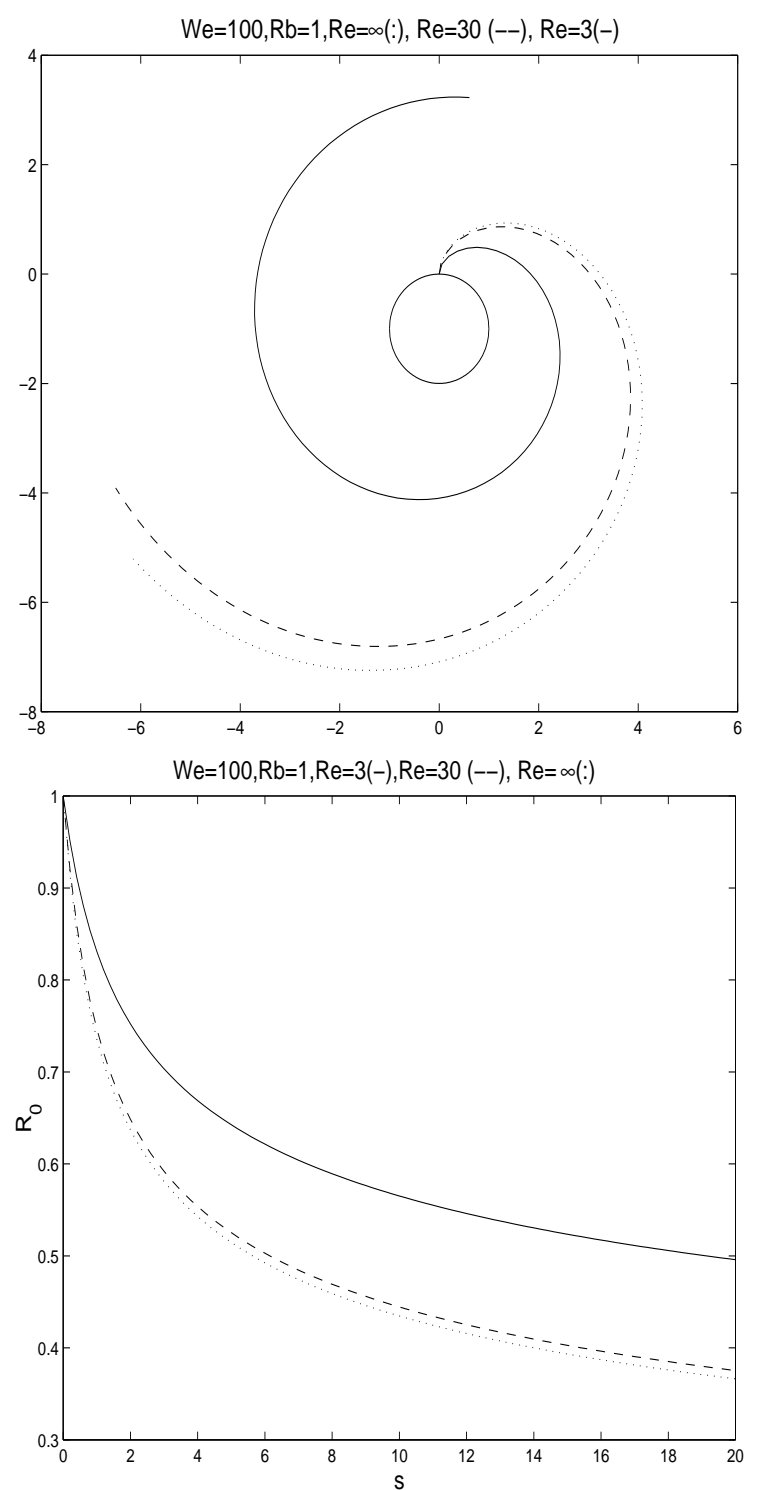

Figure 2. Top: centreline for various Re. The circle represents the container. Bottom: $R_{0}$ vs. $s$ for various $R e$. 

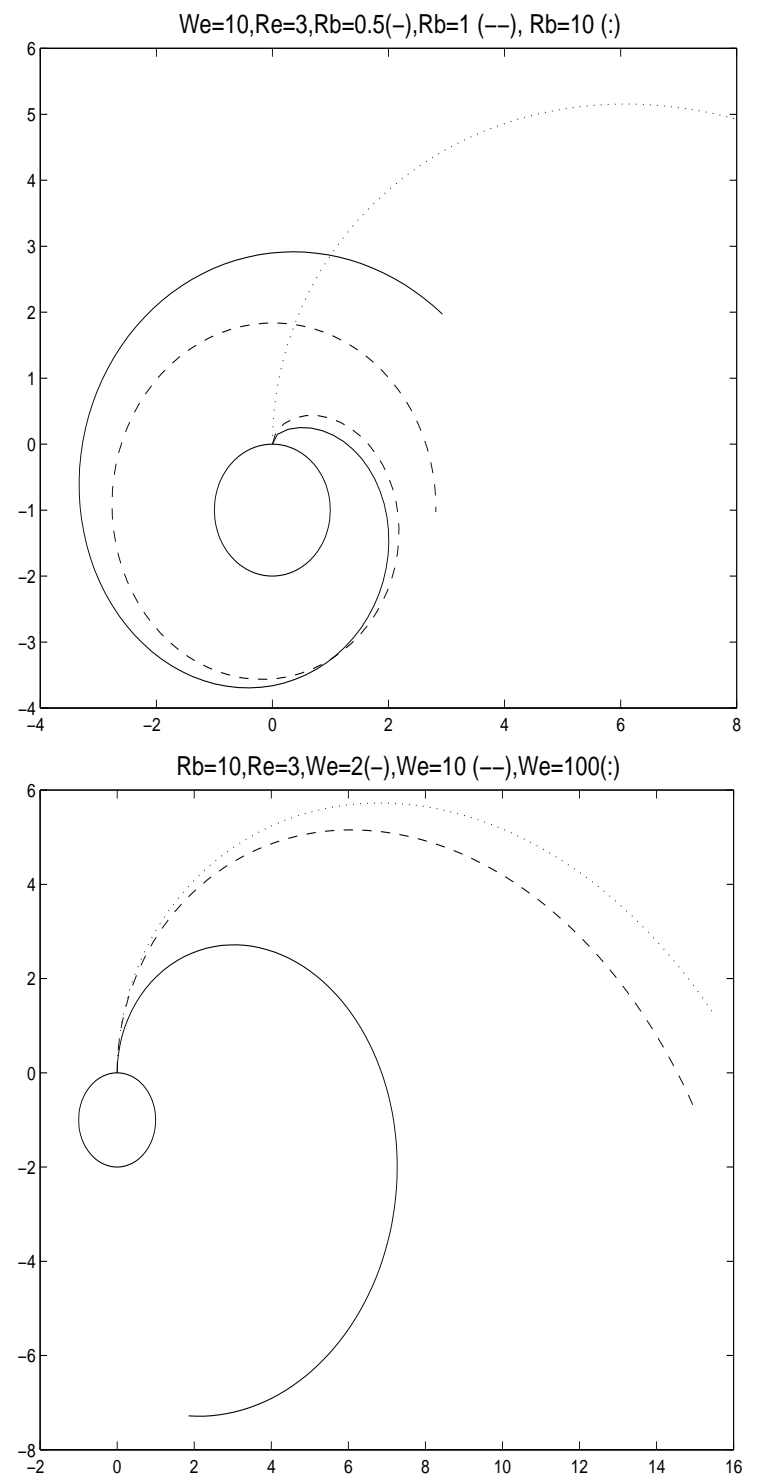

Figure 3. Top: centreline for various $R b$. Bottom: centreline for various $W e$. 

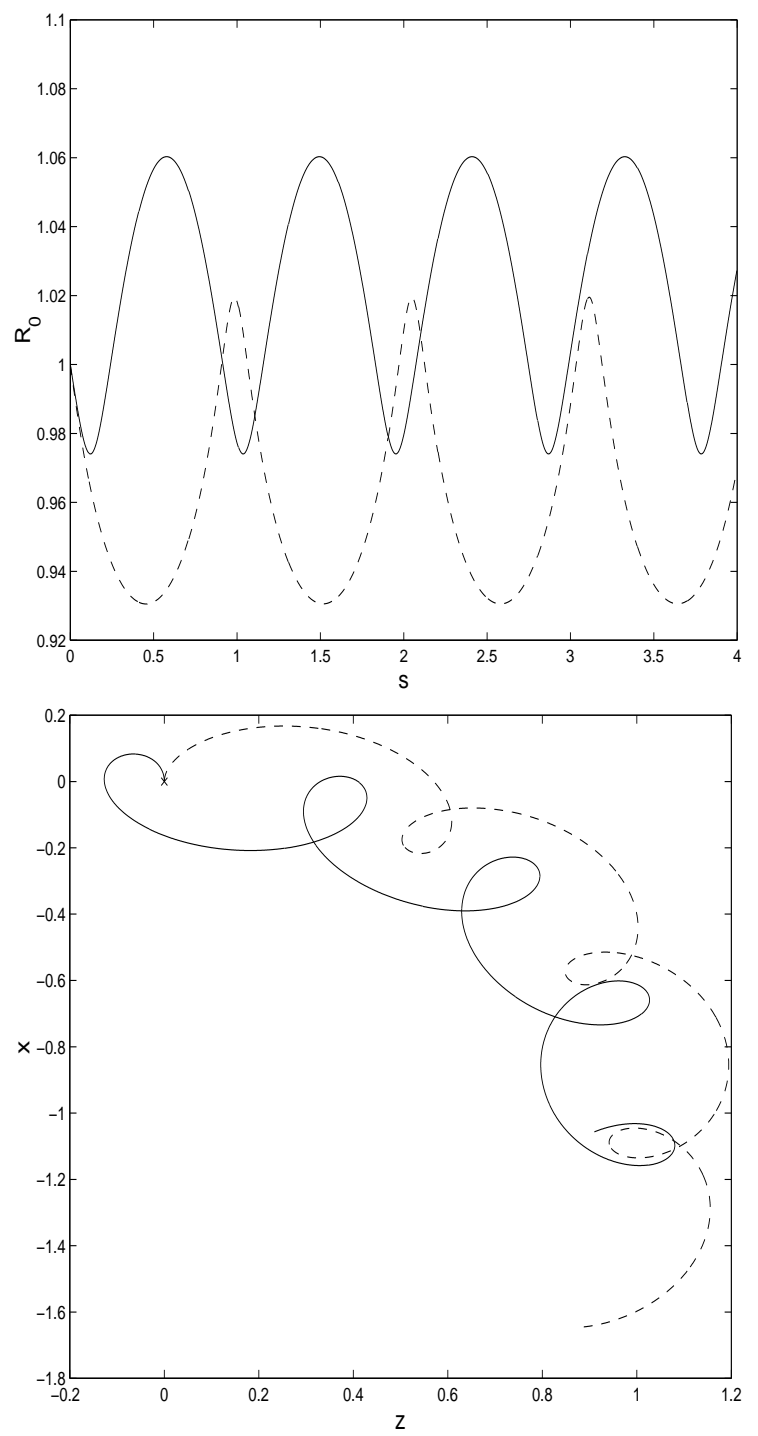

Figure 4. The radius (upper figure) and the centreline (lower figure) when the Weber number is close to one: $\mathrm{Rb}=1, \mathrm{We}=1.2$ (dashed line) and $\mathrm{Rb}=1, \mathrm{We}=0.8$ (solid line). The star marks the exit orifice. 


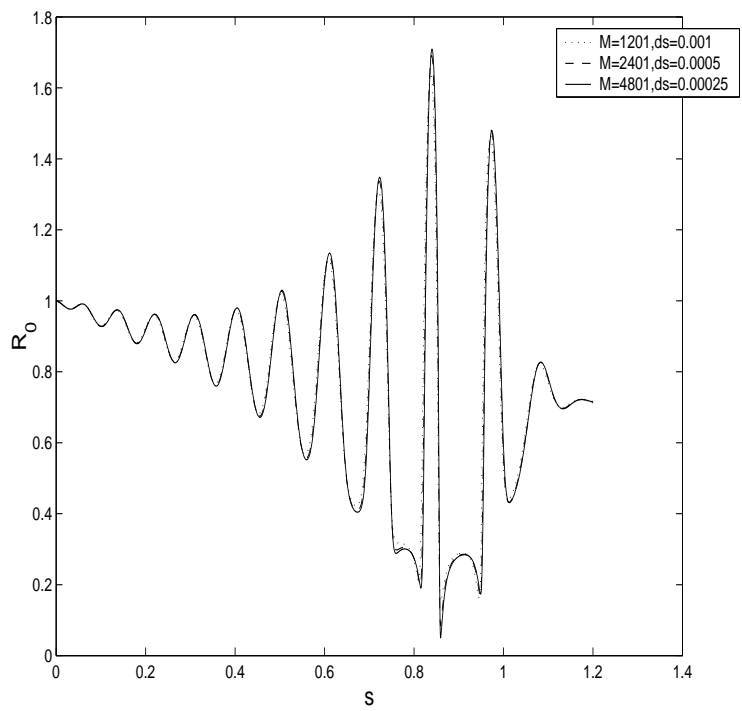

Figure 5. Accuracy check for various $d s$ and various number of mesh points $M$. The other parameters are $W e=20, R b=1, R e=3000, \delta=0.01, K=0.89, d t=10^{-6}$ and the final time $t_{f}=0.697325$. 

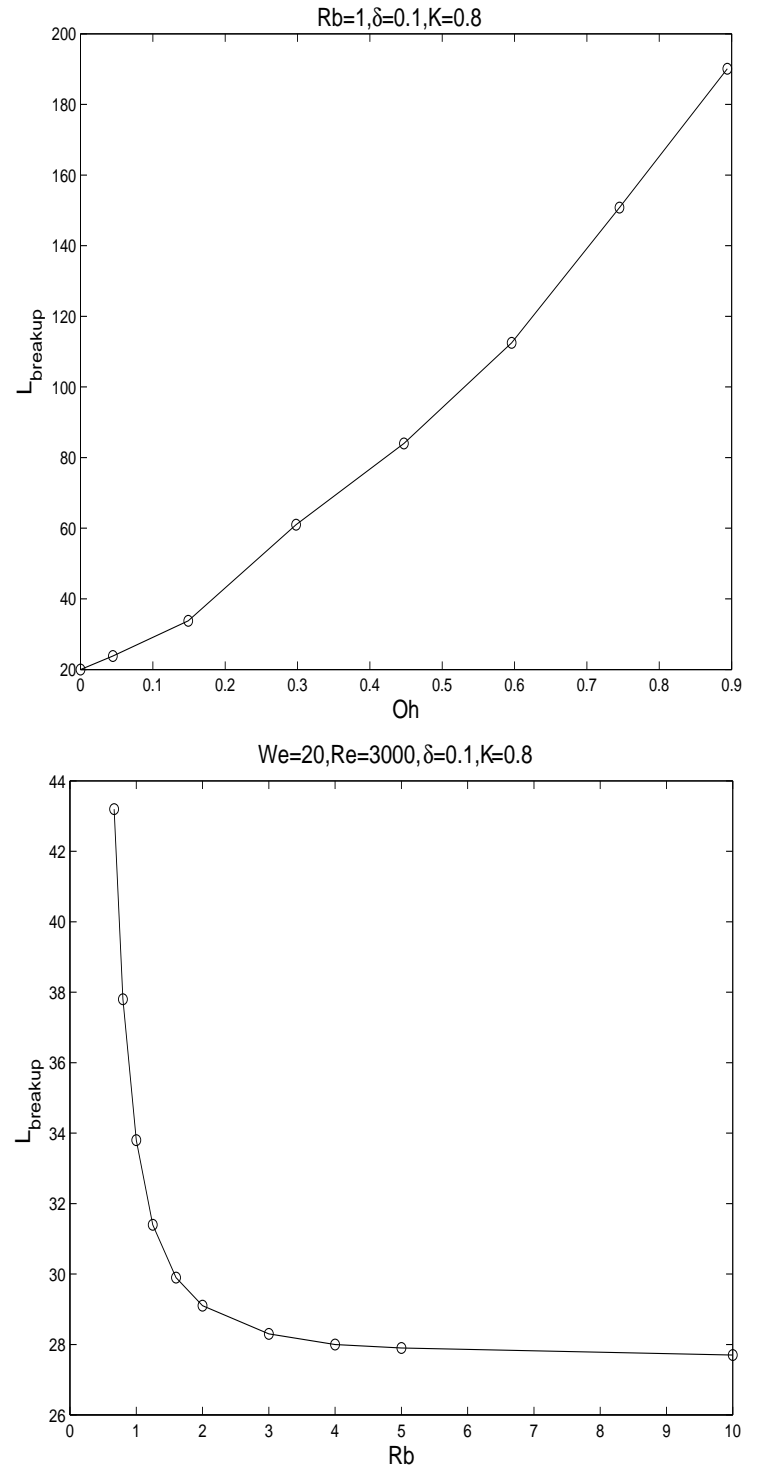

Figure 6. Top: the breakup length vs. Ohnesorge number. Here the Ohnesorge number is defined as $O h=\mu / \sqrt{\sigma a \rho}$. The breakup length increases with viscosity. Bottom:the breakup length vs. Rossby number. The breakup length increases with the rotation rate. 

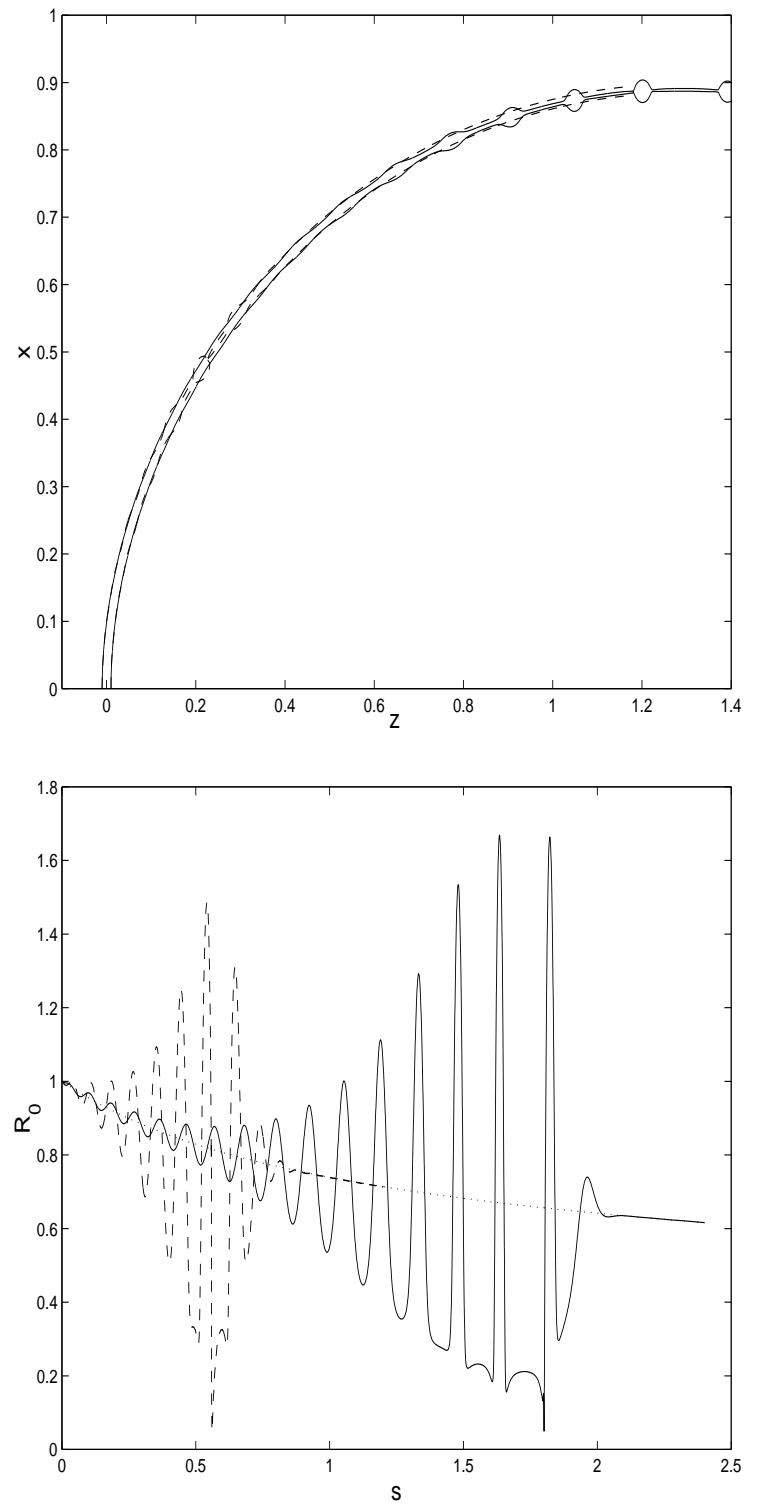

Figure 7. Top: the profiles for an inviscid jet (dashed line) and a viscous jet (solid line). The parameters are $W e=20, R b=1, \delta=0.01, R e=\infty$ or $R e=1000$. Bottom: the radius $R_{0}$ vs. $s$ for an inviscid jet (dashed line) and a viscous jet (solid line). The steady radius is also shown(dotted line). 

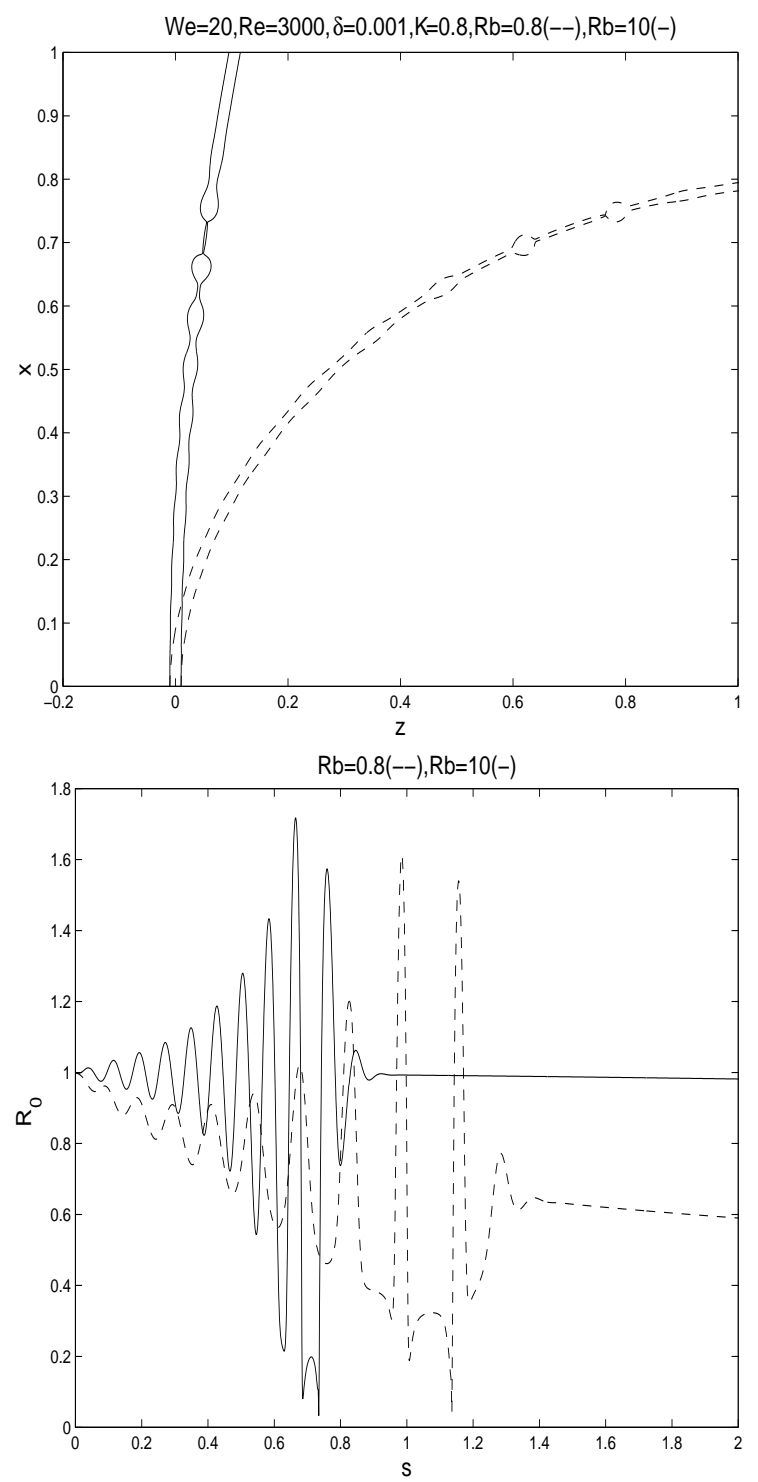

Figure 8. Top: two solutions for different Rossby numbers: $\mathrm{Rb}=0.8$ (dashed lines), $\mathrm{Rb}=10$. The other parameters are $\delta=0.01, K=0.8, R e=3000, W e=20$. Bottom: the radius $R_{0}$ against $s$. We observe that, down the jet, the wavelength of the perturbation increases with the rotation rate. 

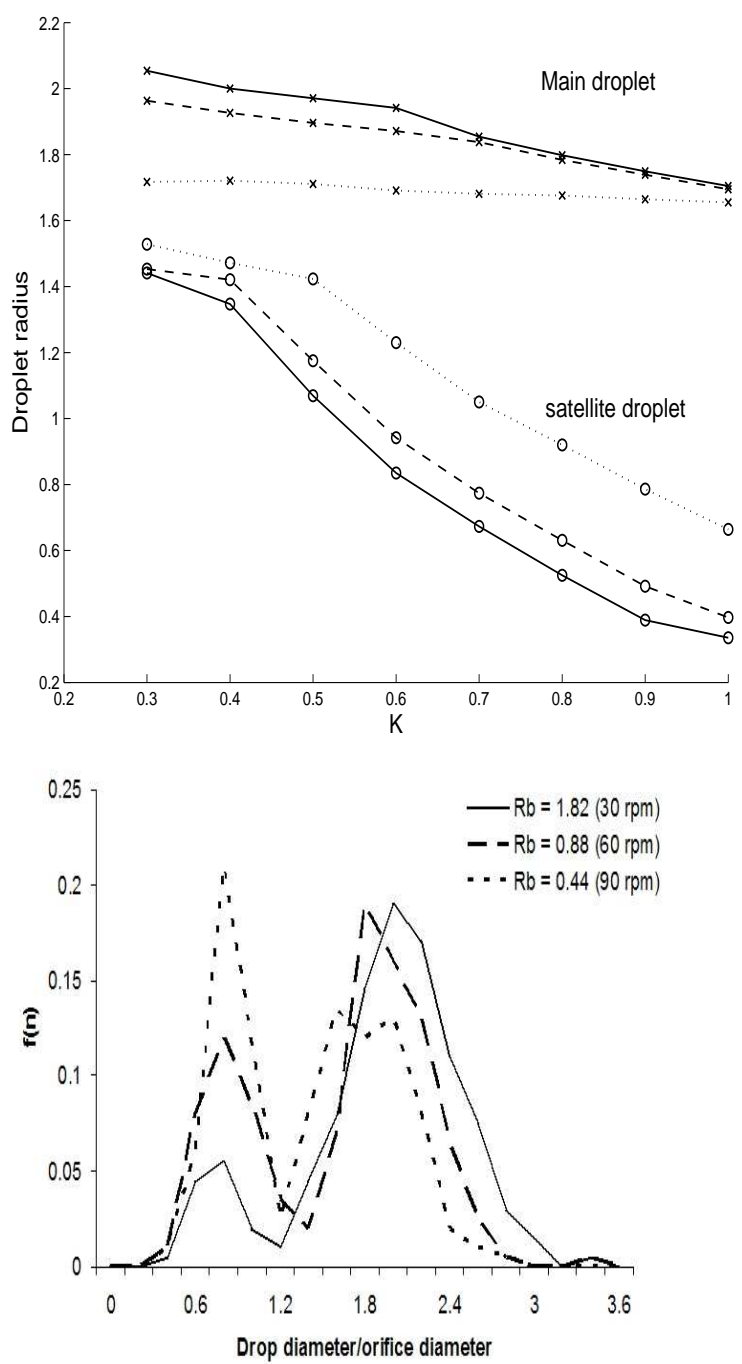

Figure 9. (a) The main drop/satellite droplet radius vs. $K$ for $\delta=0.1, W e=10, R e=3000, R b=2$ (solid line), $R b=1$ (dashed line) and $R b=0.5$ (dotted line). The satellite droplet radius increases with the rotation rate and the main drop radius decreases. (b) The figure 9 from Partridge et al. [42] showing drop size distributions for three rotational rates. 


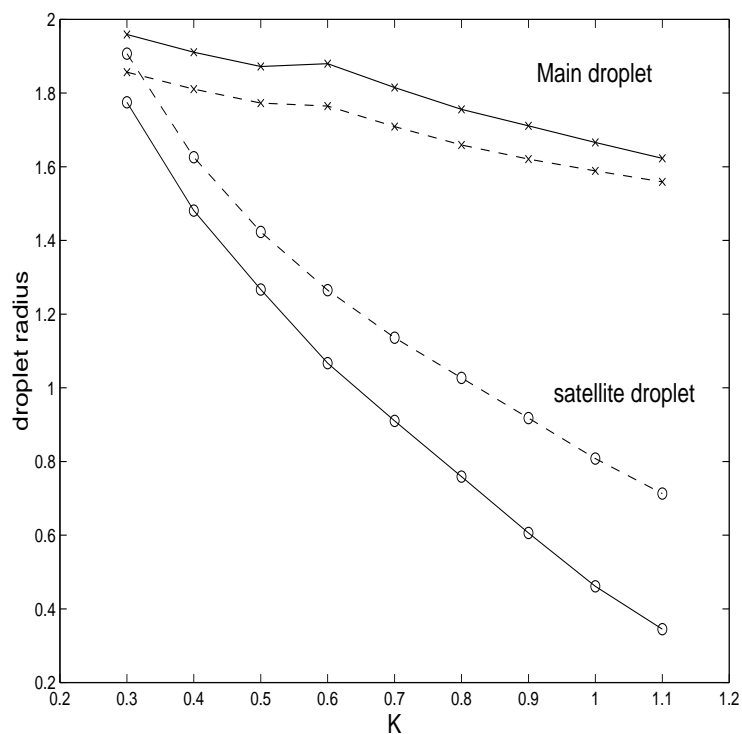

Figure 10. The main drop/satellite droplet radius vs. $K$ for $\delta=0.1, W e=100, R b=1, R e=3000$ (solid line) or $R e=300000$ (dashed line). The main drop radius increases with the viscosity and the satellite droplet radius decreases.

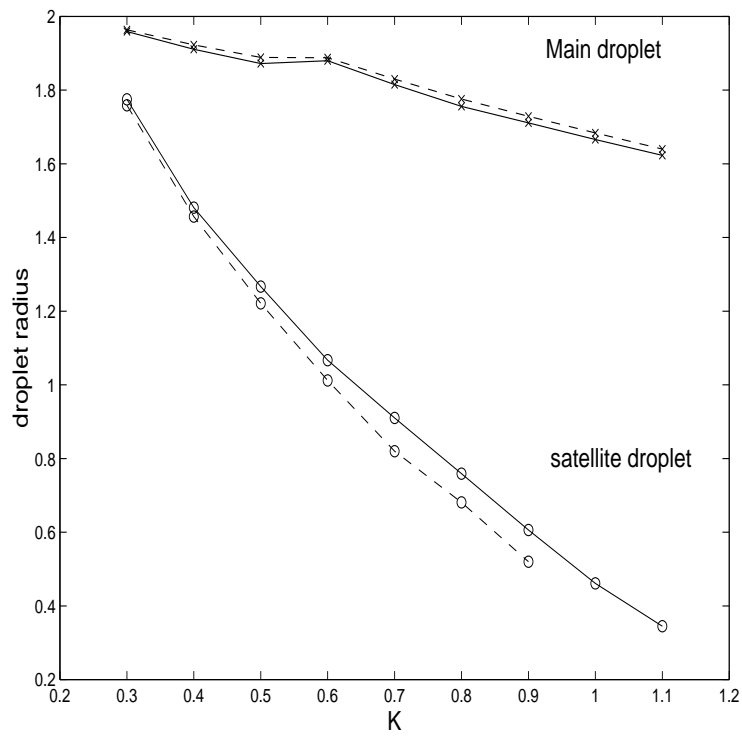

Figure 11. The main drop/satellite droplet radius vs. $K$ for $\delta=0.1, R b=1, R e=3000, W e=100$ (solid line) or $W e=20$ (dashed line). In this case, the satellite droplet radius seems to increase with the Weber number and the main drop radius to decrease. 


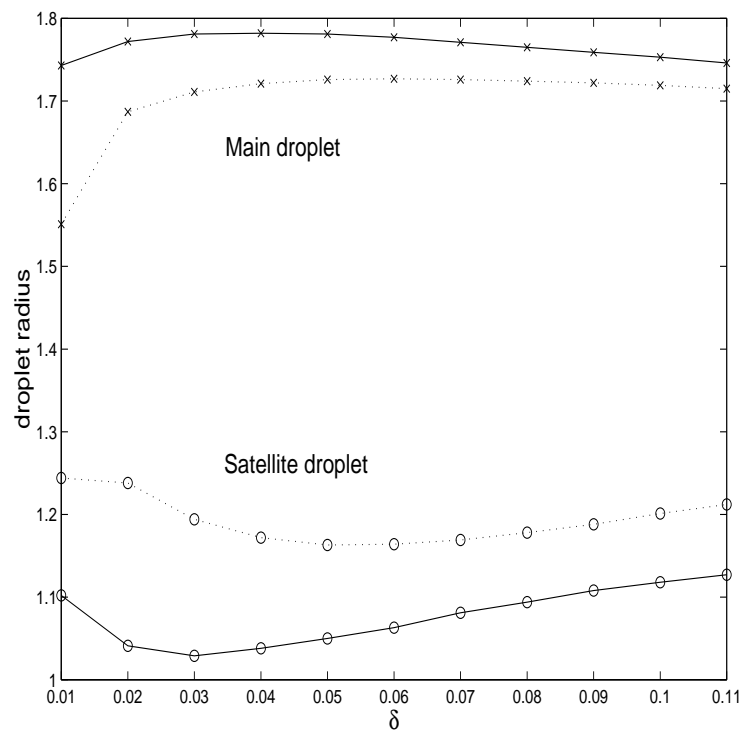

Figure 12. Droplet radii vs. disturbance amplitude $\delta$ for $W e=100, R b=1.2, R e=30000, K=0.7$ (dotted line) and $W e=50, R b=1, R e=30000, K=0.7$ (solid line). The main droplet radius and the satellite radius are shown in both cases. 


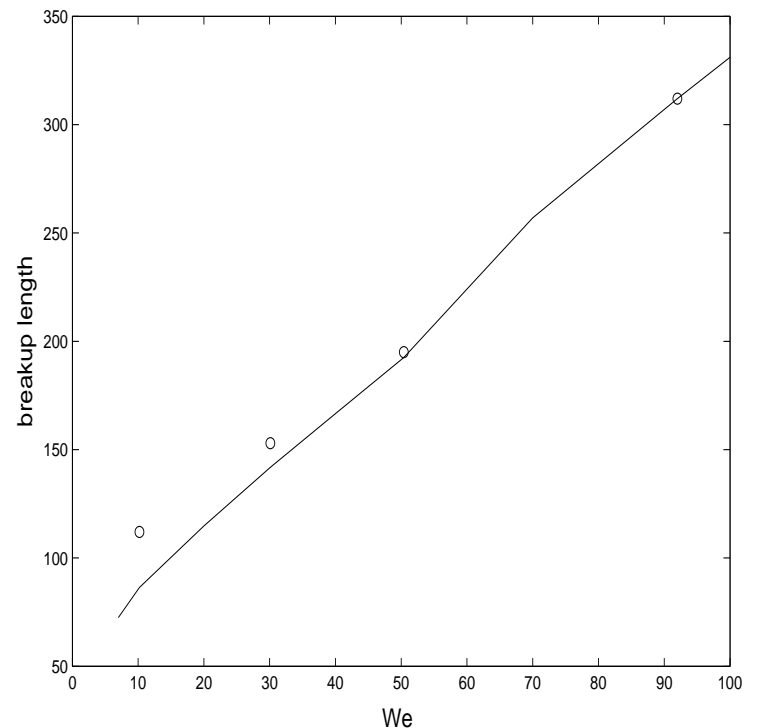

Figure 13. Experimental breakup length (circles) compared with the numerical breakup length(solid line) against the Weber number. The magnitude of the disturbance $\delta$ was chosen so that the theoretical and experimental results matched exactly in the case $W e=92$ : this gave $\delta=0.0001$ for our fitting parameter. The experimental breakup length was calculated as a mean after repeating the experiments 50 times, the scattering of data being of about $5 \%$. 


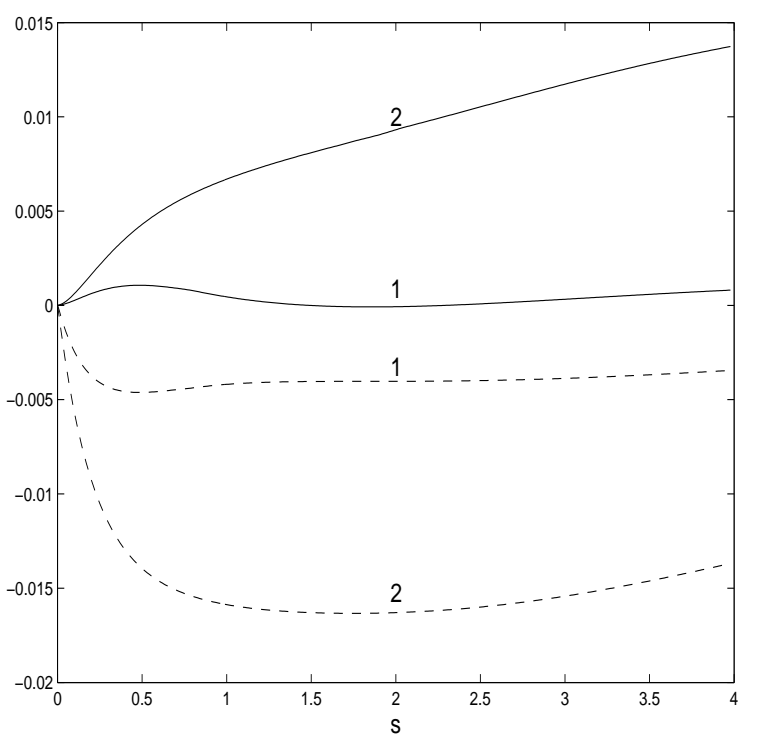

Figure 14 . The deviation $\hat{X}$ and $\hat{Z}$ of $X_{0}$ and respectively $Z_{0}$ plotted against $s$ for two different times $t$ for $\delta=0.5, W e=50, R b=1, R e=600, K=0.7$. The solid lines show $\hat{X}$ and the dashed lines show $\hat{Z}$. The lines moving away from the central axis for larger times. The times show times half-way to breakup (1) and the breakup time (2). 


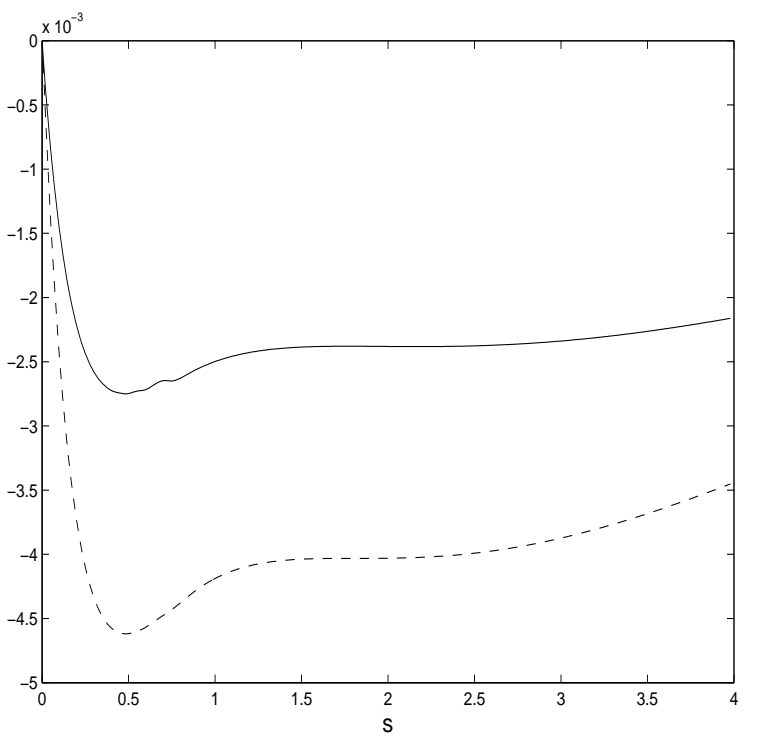

Figure 15. The deviation $\hat{Z}$ of $Z_{0}$ plotted against $s$ at the same time for two different viscosities for $\delta=0.5, W e=50, R b=1, R e=600, K=0.7$. The solid lines show $\hat{Z}$ for $R e=1500$ and the dashed lines show $\hat{Z}$ for $R e=600$. The other parameters are $\delta=0.5, W e=50, R b=1, K=0.7$. The behaviour of $\hat{X}$ is simillar. 
parauengn_visc_1oct_afterproof.tex; 20/11/2006; $12: 44 ;$ p.38 